\title{
APPLYING NEURAL NETWORKS FOR TIRE PRESSURE MONITORING SYSTEMS
}

\author{
A Thesis \\ presented to \\ the Faculty of California Polytechnic State University, \\ San Luis Obispo
}

In Partial Fulfillment

of the Requirements for the Degree

Master of Science in Mechanical Engineering

by

Alex Kost

March 2018 
(C) 2018

Alex Kost

ALL RIGHTS RESERVED 


\section{COMMITTEE MEMBERSHIP}

TITLE:

AUTHOR:

DATE SUBMITTED:
Applying Neural Networks for Tire Pressure Monitoring Systems

Alex Kost

March 2018

COMMITTEE CHAIR: Mohammad Noori, Ph.D.

Professor of Mechanical Engineering

COMMITTEE MEMBER: Xiao-Hua Yu, Ph.D.

Professor of Electrical Engineering

COMMITTEE MEMBER: Franz Kurfess, Ph.D.

Professor of Computer Science

COMMITTEE MEMBER: William Murray, Ph.D.

Professor of Mechanical Engineering 


\begin{abstract}
Applying Neural Networks for Tire Pressure Monitoring Systems
\end{abstract}

\begin{abstract}
Alex Kost
A proof-of-concept indirect tire-pressure monitoring system is developed using neural networks to identify the tire pressure of a vehicle tire. A quarter-car model was developed with Matlab and Simulink to generate simulated accelerometer output data. Simulation data are used to train and evaluate a recurrent neural network with long short-term memory blocks (RNN-LSTM) and a convolutional neural network (CNN) developed in Python with Tensorflow. Bayesian Optimization via SigOpt was used to optimize training and model parameters. The predictive accuracy and training speed of the two models with various parameters are compared. Finally, future work and improvements are discussed.
\end{abstract}




\section{ACKNOWLEDGMENTS}

Although I technically started work on this thesis in Fall 2015, I did not begin to make meaningful progress until Winter 2016. I procrastinated and pushed this off for so long that many friends began to ask me if - not when-I would graduate.

So to my thesis adviser Dr. Mohammad Noori, thank you for your patience, support, and kindness throughout this work. I could not have asked for a more supportive and understanding adviser.

To my friend Han Tran, who encouraged me to work with Professor Noori on a very non-mechanical thesis topic and pushed me to perform to the best of my capabilities. You are the reason for much of my success.

To my fraternity; SPD TID.

And finally to my family, who never failed to remind me that I must graduate by June 2017 “or else." I didn't and everything turned out ok. 


\section{TABLE OF CONTENTS}

Page

LIST OF TABLES . . . . . . . . . . . . . . . . . . viii

LIST OF FIGURES ..................... . . ix CHAPTER

1 Introduction . . . . . . . . . . . . . . . . . . 1

1.1 Background . . . . . . . . . . . . . . . . . . . 1

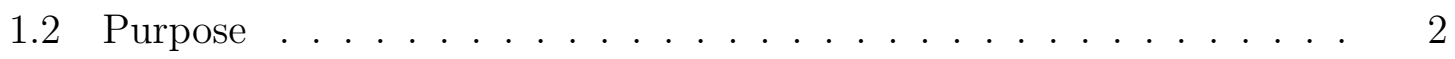

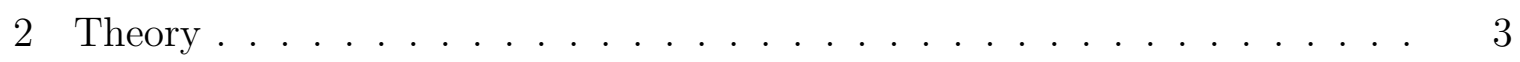

2.1 Suspension and Tires . . . . . . . . . . . . . . . 3

2.2 TPMS Architectures $\ldots \ldots \ldots \ldots \ldots \ldots \ldots \ldots$

2.3 Artificial Neural Networks . . . . . . . . . . . . . . 5

3 Work . . . . . . . . . . . . . . . . . . . . 10

3.1 Collecting Data . . . . . . . . . . . . . . . . 10

3.2 Building the Algorithm . . . . . . . . . . . . . . . 12

3.2 .1 Specifications . . . . . . . . . . . . . . . 12

3.2 .2 Development . . . . . . . . . . . . . . . . . 13

3.2 .3 Tuning . . . . . . . . . . . . . . . . . . 15

4 Results and Conclusions . . . . . . . . . . . . . . . 16

4.1 Initial Results . . . . . . . . . . . . . . . . . 16

4.2 Final Results and Discussion . . . . . . . . . . . . . . 17

4.3 Future Work . . . . . . . . . . . . . . . . . . . . . 19



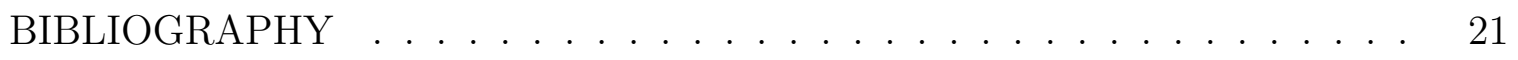




\section{APPENDICES}

A Derivations of Simulation Input Parameters . . . . . . . . . . . . . 25

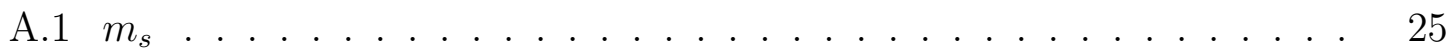

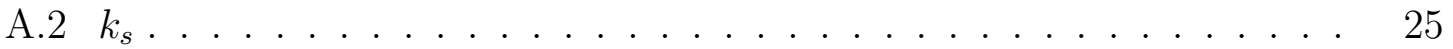

A.3 $m_{u} \ldots \ldots \ldots \ldots \ldots \ldots$

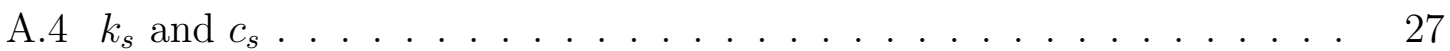

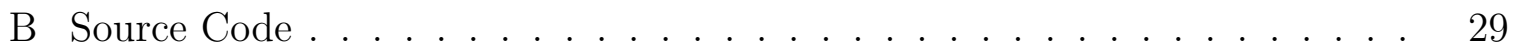

B.1 cnn_model.py . . . . . . . . . . . . . . . . . 29

B.2 rnn_model.py . . . . . . . . . . . . . . . . . . 34

B.3 data processor.py . . . . . . . . . . . . . . . . . . . 39

B.4 train.py ............................. 44

B.5 tune.py .............................. 52

B.6 main.m. . . . . . . . . . . . . . . . . 59

B.7 QuarterModelSimulation.m ............... 62

B.8 CalculateSuspensionStiffnessDamping.m . . . . . . . . 64

B.9 CalculateTireStiffness.m . . . . . . . . . . . . 65

B.10 SimFunc.m . . . . . . . . . . . . . . 66

B.11 QuarterModelMatrix.slx . . . . . . . . . . . 68 


\section{LIST OF TABLES}

Table

3.2 Inflation classifications, pressures, and labels. . . . . . . . . . 11

3.1 Simulation input variables. . . . . . . . . . . . . . . 11

3.3 Example of simulated data: Sim_35.5psi_0.75m.csv. . . . . . . . 11

3.4 Parameters optimized via SigOpt Bayesian optimization. * denotes that the parameter is related to Adam optimization strategy . . . .

4.1 Final hyperparameters chosen for both models. . . . . . . . . . . 17 


\section{LIST OF FIGURES}

Figure

2.1 A free-body diagram of the quarter-car model. Taken from Jazar et

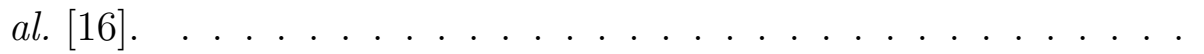

2.2 (a) Grid Search vs. (b) Bayesian Optimization techniques for tuning, where each yellow dot indicates a model evaluation. Notice that grid searches could be searching along a potentially-coarse grid, whereas Bayesian optimization techniques test any possible combination within the space and intelligently suggests combinations to reach optimal solutions with fewer evaluations. . . . . . . . . . .

2.3 A visual representation of a single block in a recurrent neural network (RNN). Taken from [29]. . . . . . . . . . . . . 7

2.4 Visualization of a 5x5 filter convolving around an input volume and producing an output. Taken from [5]. . . . . . . . . . . . 7

2.5 Visualization of a fully-connected layer. Taken from Hollemans et al. [13]. . . . . . . . . . . . . . . . . . 8

3.1 Sprung mass vs. time for $p_{u}=26,32,38$ psi for step size $y=2 \mathrm{~m}$. $\quad 12$

3.2 (a) RNN-LSTM and (b) CNN model visual graphs as created by

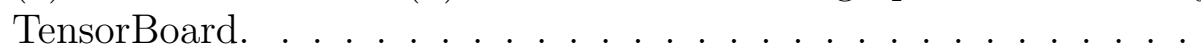

4.1 RNN-LSTM: Training classification accuracy for various Adam optimization strategy optimization parameters learning_rate, beta_1, beta_2, and epsilon. . . . . . . . . . . . . 16

4.2 Classification Accuracy During Training . . . . . . . . . . . . 18

A.1 Static stiffness vs. inflation pressure for a radial-ply car tire. [30] . . 26

B.1 QuarterModelMatrix.slx .............. 68 
Chapter 1

INTRODUCTION

\section{$1.1 \quad$ Background}

It is difficult to understate how important properly pressurized tires are to the performance and safety of a vehicle and its operator, respectively. The National Highway Traffic Safety Administration (NHTSA) estimates that 11,000 tire-related crashes occur annually in the US, with 200 people estimated to be killed in these crashes [28]. Furthermore, under-inflated tires contribute to the following performance issues when driving [37]:

1. Poor fuel economy, wasting an estimated 3.5 million gallons daily and costing drivers as much as 11 cents per gallon in the US.

2. Longer stopping distances and sluggish/ineffective handling, resulting in more dangerous driving conditions.

3. Faster tire wear, reducing the average life of a tire by 4,700 miles.

Tire-pressure monitoring systems (TPMS) became federally mandated in 2000 by the Transportation Recall Enhancement, Accountability, and Documentation Act, where legislators ruled to "require a warning system in new motor vehicles to indicate to the operator when a tire is significantly under inflated" [38]. More specifically, all motor vehicles must have a system that is capable of detecting when one or more of the vehicle's tires, up to all four tires, is $25 \%$ or more below the manufacturer's recommended inflation pressure or a minimum activation pressure specified in the standard, whichever is higher [25]. Nonetheless, a study performed in April 2009 showed that $45 \%$ of TPMS-enabled vehicles still have under-inflated tires [26].

Therefore, for obvious moral and legal reasons, it is imperative that drivers know that

their tires are inflated properly. It is in the individual's and society's best interests 
to improve safety, performance, and savings while on the road.

\subsection{Purpose}

The most commonly used TPMS in vehicles today is a simple pressure sensor mounted within the tire to directly measure the pressure of the air within the tire. When the integrated battery dies on these sensors, the sensors must be replaced manually. Time, money, and labor are spent to replace this simple sensor. It would be advantageous if the TPMS architecture was created such that maintenance and repair were not needed.

As advancements in machine learning and deep learning techniques continue, it is no longer a question of how or why to apply these techniques, but where to apply them. In this work, a proof-of-concept TPMS architecture is suggested that uses accelerometer data and deep learning algorithms to determine whether the tires on a vehicle are under, over, or nominally inflated. This work is comprises four chapters:

1. Chapter 1 introduces the legal and moral motivations behind TPMSs in today's vehicles. This chapter also outlines the content of this work.

2. Chapter 2 serves as a literature review for this work. Three specific fields of study are defined: a mechanical review of automotive suspension systems and tires, current TPMS frameworks and sensing capability, and current technologies and research in artificial neural networks.

3. Chapter 3 presents the work done to create a proof-of-concept classifier using simulated data. The simulated model and its limitations are discussed, as well as the architecture and modifications done on the artificial neural network.

4. Chapter 4 compares the final performance of the implemented classifiers and concludes this work with a discussion on the meaning and limitations of the results. Suggestions for future work are also made. 
Chapter 2

\section{THEORY}

\subsection{Suspension and Tires}

Vehicle suspensions and tires are designed to optimize the traction, ride comfort, handling, and fuel consumption of the vehicle. The suspension links the wheelstires mated to rims - to the vehicle chassis and allows relative motion, while the tire transfers energy between the vehicle and the road to allow the vehicle to move [16]. Together, the suspension and tires are the defining aspects of a vehicle's combined stiffness and damping coefficients.

A simplified representation of a vehicle suspension system is used in this work. Known as a Quarter Car Model, the representation has only one degree-of-freedom and can only move vertically. The vehicle is rigid; only vibrations transferred from the ground to the tires, axles, and suspension systems are considered. This representation also does not consider any forces or reactions due to the geometry of the vehicle; it is only looking at a single wheel on this "vehicle." The representation is presented in Figure 2.1 [16].

The unsprung mass $m_{u}$ refers to all masses that are attached to and not supported by the spring, such as the wheels, axles, or brakes. In this representation, the unsprung mass is the weight of the tire and the weight of the air of the tire. In an actual vehicle, suspension stiffness and damping values $k_{s}$ and $c_{s}$ are functions of suspension type, tire geometry, tire pressure, vehicle geometry, and vehicle weight. These values should be constant in vehicles without active suspension systems, so the only changing parameter in this model is the unsprung mass's stiffness $k_{u}$. Any damping in parallel with $k_{u}$ is negligible with respect to $c_{u}$ and is thus not included in the representation. 




Figure 2.1: A free-body diagram of the quarter-car model. Taken from Jazar et al. [16].

\subsection{TPMS Architectures}

The NHTSA provides vehicle manufacturers three ways to comply with the law: direct, indirect, and hybrid TPMS [27]. Direct TPMS consists normally of pressure sensors located inside each wheel to directly measure the pressure in each tire. Indirect TPMS compares speed data collected from vehicle's anti-lock braking system wheel speed sensors to compare rotational speeds of tires against one another to determine the pressure. Direct systems are more accurate and precise, whereas indirect systems are less hardware-dependent and more robust for each vehicle. The NHTSA leaves the definition of a hybrid TPMS purposefully vague and suggests such a system would use a combination of direct and indirect methods to fulfill the regulatory requirements. Although direct TPMS dominates the method today, indirect TPMS is expected to become the dominant TPMS in the coming years.

A note should be made that not all direct and indirect TPSM are created equal: individual features differ from system to system. As shown in Kubba and Jiang [21], various direct TPMS systems use different power sources and sensing solutions.

Research of indirect TPMS frameworks has grown and continues to grow because of their perceived advantages over direct TPMS as computing power increases. For 
example, Persson, Gustafsson, and Drev [31] presented in 2002 an indirect TPMS combining vibration and wheel radius analyses was able to detect pressure losses larger than $15 \%$ in one, two, three, or four tires and identify the underinflated tire within 1 minute.

\subsection{Artificial Neural Networks}

An artificial neural network (ANN) is a machine learning algorithm used to solve advanced non-linear problems such as handwriting or speech recognition. Neural networks connect computational nodes together to form a singular "network," where each computational node is performing a calculation on its input and outputting the result to all outgoing connections. The output of a node can be the input to at least one other node or to many other nodes. Outputs can be scaled and biased by weights and biases respectively; think the canonical linear function $y=m x+b$, where $y$ is the original output, $m$ is the weight, $x$ is the new output, and $b$ is the bias. Often, activation functions are added to the networks; these further define the output with a linear or non-linear function. As shown by Ramachandran et. al [34], the most commonly used activation function in deep learning projects is the rectified linear unit (ReLU). In summary, interconnected computational nodes perform linear and non-linear operations on inputs.

At first, all ANN models do not perform well because the weights and biases are not tuned; that is, the model is not trained. Neural networks can learn a hierarchical feature representation from raw data automatically [40]; that is, they "learn" or can be trained through example. In this work, we train our models via supervised learning - that is, with labeled training data - and compare the model's predictions to the actual labels. By repeatedly minimizing the error between prediction and truth, the model updates the trainable parameters and its accuracy improves. This updating is based on minimizing a cost (generally inversely proportional to accuracy) via some optimization strategy. Gradient Descent strategies are often implemented; in this work, the Adaptive Moment Estimation (Adam) strategy is applied. Adam computes adaptive learning rates for each parameter and takes advantage of the 
idea of momentum to more quickly converge on the global minima with reduced oscillation [20].

Furthermore, models hyperparameters can be tuned such that they can more quickly be trained and perform more optimally. Grid search tuning is a standard method where an exponentially large grid of possible hyperparameter combinations is systematically searched. Alternatively, Bayesian Optimization tuning promises a more intelligently search by learning from prior hyperparameter combinations and their results to intelligently suggest better combinations [6]. Grid searches are exponentially expensive whereas Bayesian optimization are only linearly expensive, as visualized in Figure 2.2. In this work, the software-as-a-service product SigOpt is applied to perform Bayesian optimization techniques for quick, intelligent tuning.

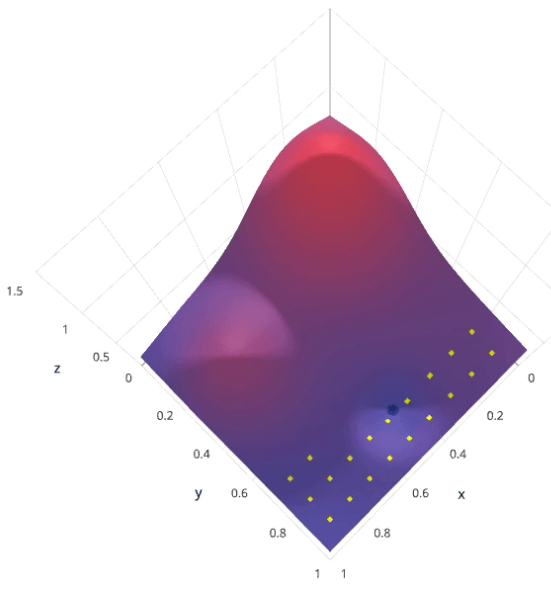

(a)

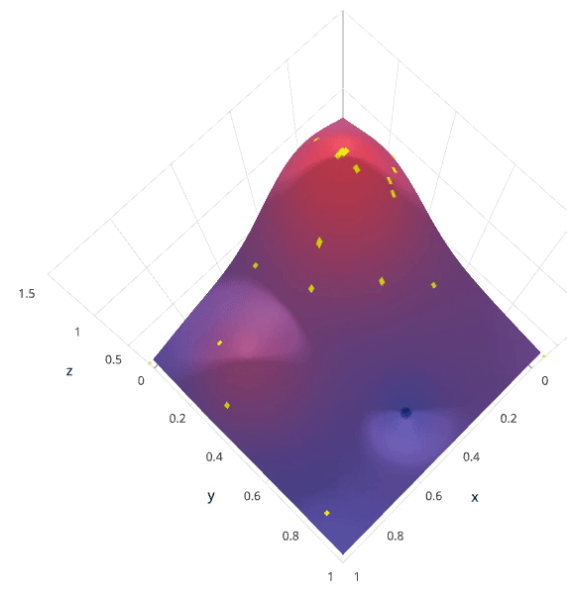

(b)

Figure 2.2: (a) Grid Search vs. (b) Bayesian Optimization techniques for tuning, where each yellow dot indicates a model evaluation. Notice that grid searches could be searching along a potentially-coarse grid, whereas Bayesian optimization techniques test any possible combination within the space and intelligently suggests combinations to reach optimal solutions with fewer evaluations.

The type of input data generally defines the type of ANN to be used; in this case, the models are interpreting time series data. As defined by Dorffner [8], a time series is a sequence of vectors depending on time $t$ such that $\vec{x}(t), t=0,1,2$, and so on. The components of $\vec{x}$ at each time $t$ (referred to as datapoints in this work) are distinct from one another but are not informative enough to extrapolate meaningful 
information from the time series; instead, each datapoint in a time series must be analyzed in relation to the rest of the time series. We discuss two major model types for interpreting time series data below: the recurrent neural network (RNN) and convolutional neural network (CNN).

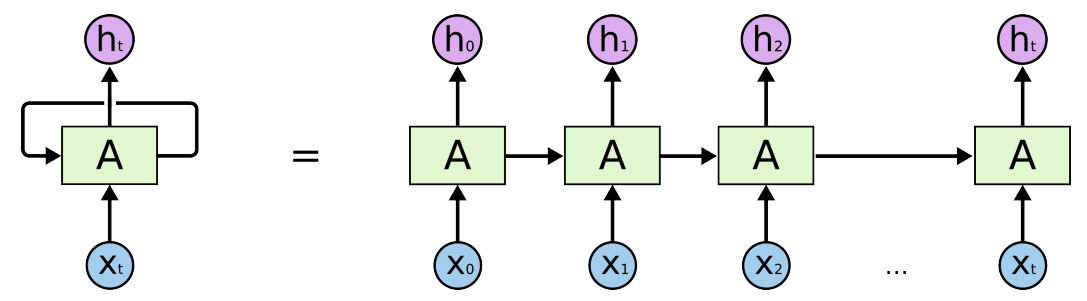

Figure 2.3: A visual representation of a single block in a recurrent neural network (RNN). Taken from [29].

Recurrent neural networks (RNNs) interpret time-series data successfully by adding feedback loops to the standard ANN network architecture [22] [9]. Some RNNs use more complex computational nodes known as long short-term memory (LSTM) blocks to mitigate an issue common in RNNs known as the vanishing gradient problem [9].

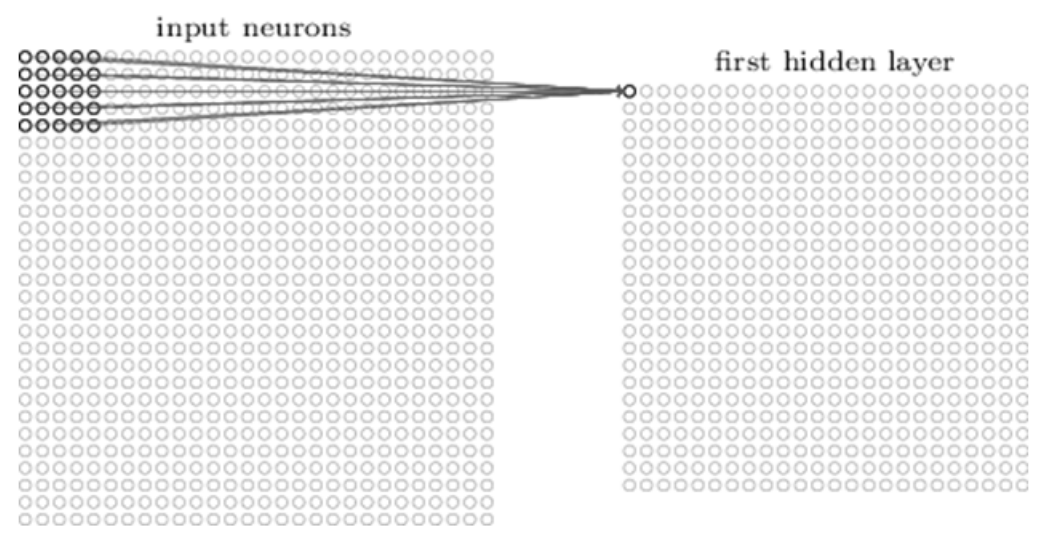

Figure 2.4: Visualization of a $5 \times 5$ filter convolving around an input volume and producing an output. Taken from [5].

Convolutional neural networks (CNNs) interpret clusters of datapoints (e.g. timeseries, images, sentences, sound recordings, so on) together to preserve spatial or temporal relationships. CNNs apply kernels or filters - i.e. a weight matrices - to recognize and extract features or patterns [19].

The first few layers of a typical ANN act as feature extractors; that is, they are responsible for extracting meaningful information from the input data. For example, 
RNNs build an internal memory and CNNs use pattern matching. This meaningful information is then fed into a classifier. Classifiers are generally fully-connected layers (each node is connected to one another; see Figure 2.5) with $n$ outputs, where $n$ is the number of classes in the input data.

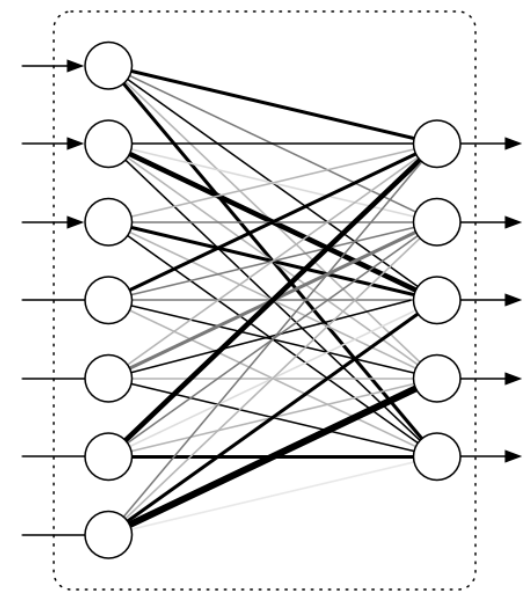

Figure 2.5: Visualization of a fully-connected layer. Taken from Hollemans et al. [13].

ANNs have been applied in the automotive industry for decades. In 1990, Wiggins presented a neural network that could identify engine faults based on the vehicle's engine controller data [39]. Neural networks were used to control the air-to-fuel ratio in fuel injection systems as shown by Alippi et al. in 2003 [2]. More recently, ANNs have driven advances in automated vehicle control ("self-driving") that can detect, identify, and respond to objects and pedestrians on the road in real time. While Tesla, Mercedes-Benz, and BMW were first introduce these features to consumer vehicles, the technology is becoming increasingly ubiquitous [17]. A NHTSA investigation conducted in January 2017 found crash rates Tesla crash rates have dropped by almost 40\% since enabling self-driving capabilities in 2015 [12].

Applying ANNs to automobiles requires dedicated software and hardware on the vehicle. Unlike data centers, portable implementations are limited primarily by the size, energy, and computational power of the device they are operating on [33]. Size is generally not a constraint for automotive manufacturers. Energy and computational power are proportional: therefore, research has been focused on improving microprocessing architectures to minimize energy draw (hardware) or improving the efficiency 
of the algorithm to reduce computational load (software). With respect to hardware, in February 2016, researchers presented a convolutional neural network accelerator chip that uses 10X less power and requires 4.7x fewer DRAM accesses per pixel than a mobile GPU [4]. Similarly, with respect to software, AlphaGo, a Google project, demonstrated that integrating classification trees with neural networks significantly reduces the computational burden, making what people once thought impossible - a computer defeating a world-champion Go player in real time - possible [35]. Many more examples like these can be found. 
Chapter 3

\section{WORK}

With the desire to explore alternative indirect TPMS frameworks and inspired by deep learning is seemingly infinite applications, this work explores a deep learning framework that analyzes vehicle suspension acceleration data to classify the vehicle tires as under-inflated, nominally inflated, or over-inflated. To validate this idea, work was broken into the following sections:

1. Collecting Data. The accuracy and capability of the ANN is largely dependent on the size of our data-ANNs tend to improve when there is more data for training. In this work, data was simulated by a quarter-car model written in Matlab and Simulink. The data serves as the training, validation, and test sets for the ANN.

2. Creating the Algorithm. Using the data from the prior step, an RNN-LSTM and CNN are developed in Python with Google's open-source TensorFlow API. Tuning model and training parameters are done using Bayesian Optimization via SigOpt.

\subsection{Collecting Data}

A Matlab model for the quarter-car representation as shown in Figure 2.1 was run at various tire pressures and step-sizes to generate simulated examples of a vehicle suspension system experiencing a step response (in an attempt to be analogous to a pothole or speed bump). The simulation solves the system of ordinary differential equations for every time step for the position, velocity, and accelerations of the sprung mass $m_{s}$ and unsprung mass $m_{u}$. The simulation inputs are presented below in Table 3.1 and their accompanying derivations are presented in Appendix A. 


\begin{tabular}{c|c|c} 
Table 3.2: Inflation classifications, pressures, and labels. \\
Inflation Classification & Pressure Range (psi) & Label (int) \\
\hline Under & $26-30$ & 0 \\
Nominal & $30-34$ & 1 \\
Over & $34-38$ & 2
\end{tabular}

Table 3.1: Simulation input variables.

\begin{tabular}{c|l|rl} 
Variable & Description & Value & {$[$ Units $]$} \\
\hline$p_{u}$ & Tire pressure & Varies & {$[\mathrm{psi}]$} \\
$y$ & Step size & Varies & {$[\mathrm{m}]$} \\
$m_{s}$ & Sprung mass & 277.25 & {$[\mathrm{~kg}]$} \\
$m_{u}$ & Unsprung mass & 34.69 & {$[\mathrm{~kg}]$} \\
$k_{s}$ & Sprung stiffness & 557.97 & {$[\mathrm{kPa}]$} \\
$c_{s}$ & Sprung damping & 6218.35 & {$[\mathrm{~Pa}-\mathrm{sec}]$} \\
$k_{u}$ & Unsprung stiffness & Varies & {$[\mathrm{kPa}]$} \\
$g$ & Gravity & 9.81 & {$\left[\mathrm{~m} / \mathrm{sec}^{2}\right]$}
\end{tabular}

The simulation was performed for $p_{u}=25.5,26,26.5,27, \ldots, 38.5$ and for $y=0.10,0.15,0.2, \ldots$ 2.0, generating 633 total examples. Every 1.5-second-long run is composed of 1500 data points and labeled according to the inflation classifications as defined by Table 3.2. These classifications are $10 \%$ of 32 psi, well within the $25 \%$ specification as defined by the TREAD Act. The label of the simulation and the sprung's mass acceleration $\ddot{x}_{s}$ are saved in individual .csv files to be parsed by the algorithm. An example of the generated data is presented below in Table 3.3 (note that the first row is only shown here for clarification and is not included in the raw output).

Table 3.3: Example of simulated data: Sim_35.5psi_0.75m.csv.

\begin{tabular}{c|c|c|c|c|c} 
label & $\ddot{x}_{s}, t=0.000 s$ & $\ddot{x}_{s}, t=0.001 s$ & $\ldots$ & $\ddot{x}_{s}, t=0.420 s$ & $\ddot{x}_{s}, t=0.421 s$ \\
\hline 2 & -0.00073852 & -0.00067152 & $\ldots$ & -1.3974 & -1.2822
\end{tabular}


Plots were developed of $x_{s}$ vs. time as a quick sanity check. The plots make intuitive sense-higher pressure correlates with greater stiffness, which then increases the natural frequency, slows the settling speed of the mass, and reduces the maximum amplitude. The simulation is sound.

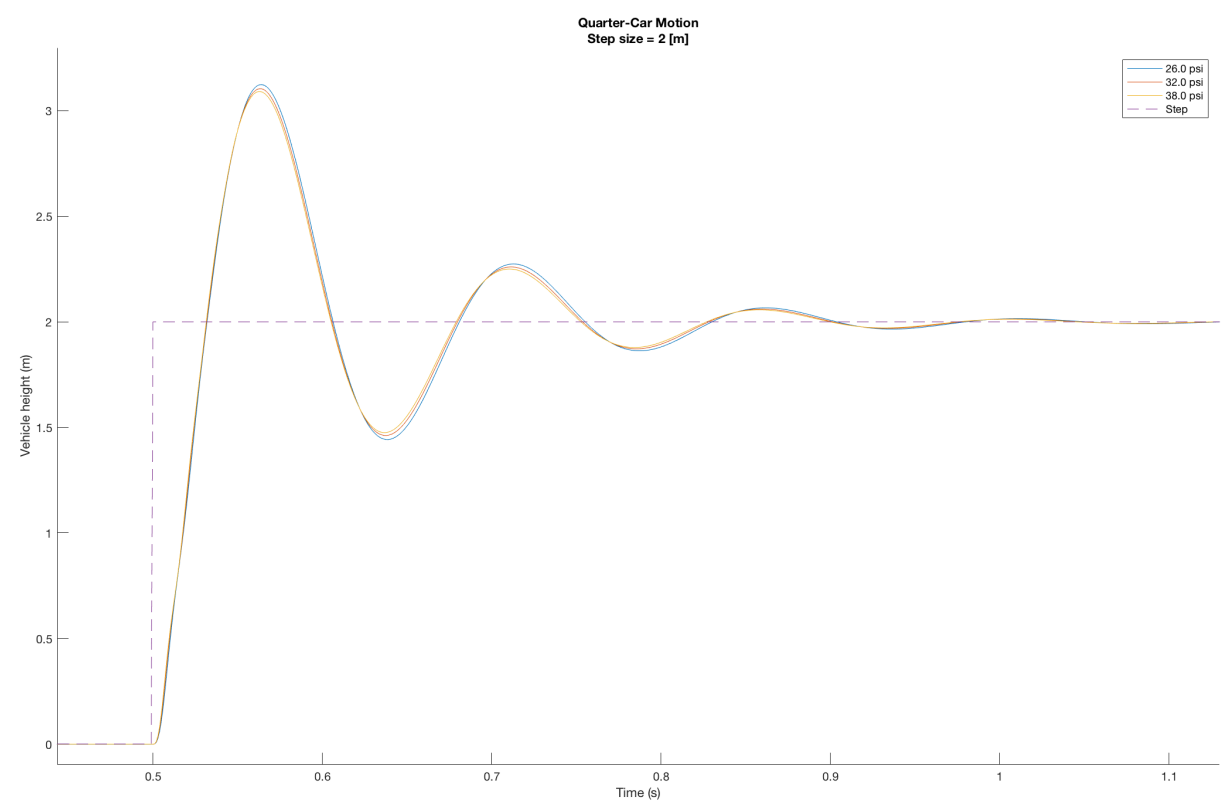

Figure 3.1: Sprung mass vs. time for $p_{u}=26,32,38$ psi for step size $y=2$ m.

\subsection{Building the Algorithm}

\subsubsection{Specifications}

For this work, TensorFlow was used to build, train, and evaluate a RNN and a CNN. Development of each neural network followed the same specifications as listed below.

1. Import the simulated data into the Python environment.

(a) The input data shall be shuffled randomly.

(b) The input data shall be split into a training set (60\%), validation set (20\%), and test set $(20 \%)$. 
2. Build the model of the neural network.

(a) The model shall be fed labeled input data and output predicted labels.

(b) The input data should be fed in batches to minimize computational load between parameter updates. Generally, the recommended starting batch size is $32[3]$

(c) The model shall prevent overfitting by applying dropout to the outputs of at least one fully-connected layer [32].

(d) Batch normalization shall be applied after various layers to reduce the internal covariate shift within the model [15].

(e) Model logits shall be converted to classification predictions using the softmax activation function.

3. Evaluate the predictive capabilities and training speed of the model.

(a) The cost shall be calculated using the cross-entropy function between the input data labels and model predictions [24].

(b) The accuracy shall be calculated by comparing the model's predicted labels to the input data labels.

(c) The training speed shall be minimized by tuning the model hyperparameters.

4. Train the model parameters.

(a) The training shall end after a predefined number of epochs and not be stopped early to observe any overfitting in the model.

(b) The training method shall minimize the batch's average cross-entropy loss using Adam Optimization strategy [20].

(c) The learning rate shall be static or exponentially decaying.

\subsubsection{Development}

The RNN-LSTM and CNN models are self-contained in RNNModel and CNNModel respectively. Both models are similar except for the feature extraction near the input 
layer of the model.

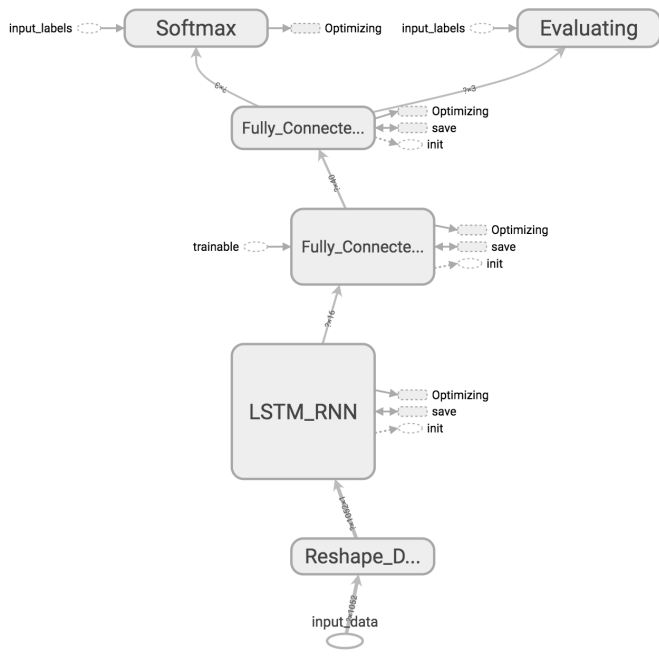

(a)



(b)

\section{Figure 3.2: (a) RNN-LSTM and (b) CNN model visual graphs as created by TensorBoard.}

A DataProcessor class was written to provide methods to scan a directory for all files and perform various preprocessing operations. In this work, DataProcessor scans the simulated data directory; generates lists of all files found across all labels; shuffles and splits the filenames across test, validation, and training sets; and loads the feature data and label data found in each files from each set into member variables to be used for training.

The training class TrainModel is the entry point to train the model. Instantiating TrainModel builds the desired model with a provided learning rate learning_rate and dropout rate dropout_rate. Calling train_model trains the model for a desired number of epochs n_epochs using feature and label data inherited from DataProcessor. Every $\frac{1}{n_{-} \text {checks }}$, the model's accuracy and cost are evaluated across the entire training and validation datasets and reported to TensorBoard for visualization. The test set accuracy is evaluated before and after training. 


\subsubsection{Tuning}

The model parameters were tuned via SigOpt to identify optimal values for various model hyperparameters. Tuning classes GridSearchTune and SigOptTune were developed to perform a grid search or connect to SigOpt to perform a Bayesian search respectively. It was estimated that a grid search over the entire model space would take over two weeks of computations per model, whereas SigOpt's more-intelligent Bayesian search strategy would take days instead. Thus, only SigOptTune was used in this work.

Two SigOpt experiments were run for each model to optimize the training speed and accuracy respectively. The parameters under investigation are listed below in Table 3.4.

Table 3.4: Parameters optimized via SigOpt Bayesian optimization. * denotes that the parameter is related to Adam optimization strategy

\begin{tabular}{l|l|c|c} 
Name & Description & RNN-LSTM & CNN \\
\hline dropout_rate & Dropout rate & $\mathrm{X}$ & $\mathrm{X}$ \\
\hline learning_rate* & Learning rate & $\mathrm{X}$ & $\mathrm{X}$ \\
\hline beta1* & 1st moment estimates exponential decay rate & $\mathrm{X}$ & $\mathrm{X}$ \\
\hline beta2* & 2nd moment estimates exponential decay rate & $\mathrm{X}$ & $\mathrm{X}$ \\
\hline epsilon* & Numerical stability constant & $\mathrm{X}$ & $\mathrm{X}$ \\
\hline num_filt_1 & Number of filters in convolutional layer & $\mathrm{X}$ \\
\hline kernel_size & Kernel size in convolutional layer & $\mathrm{X}$ \\
\hline num_fc_1 & Number of neurons in first fully-connected layer & $\mathrm{X}$ & $\mathrm{X}$ \\
\hline $\mathrm{n} \_l$ ayers & Number of hidden layers in model & $\mathrm{X}$ &
\end{tabular}

All source code is available in Appendix B. 
Chapter 4

\section{RESULTS AND CONCLUSIONS}

\subsection{Initial Results}

Tuning the Adam-specific hyperparameters gave insight in a recurring issue with the LSTM-RNN: The model would not improve in performance after 200 steps (40 epochs with batch_size $=128$ ). Figure 4.1 shows multiple training curves with various values for learning_rate, beta_1, beta_2, and epsilon. where the cross-validation accuracy would remain at $33.3 \%$, or the same accuracy as randomly guessing.

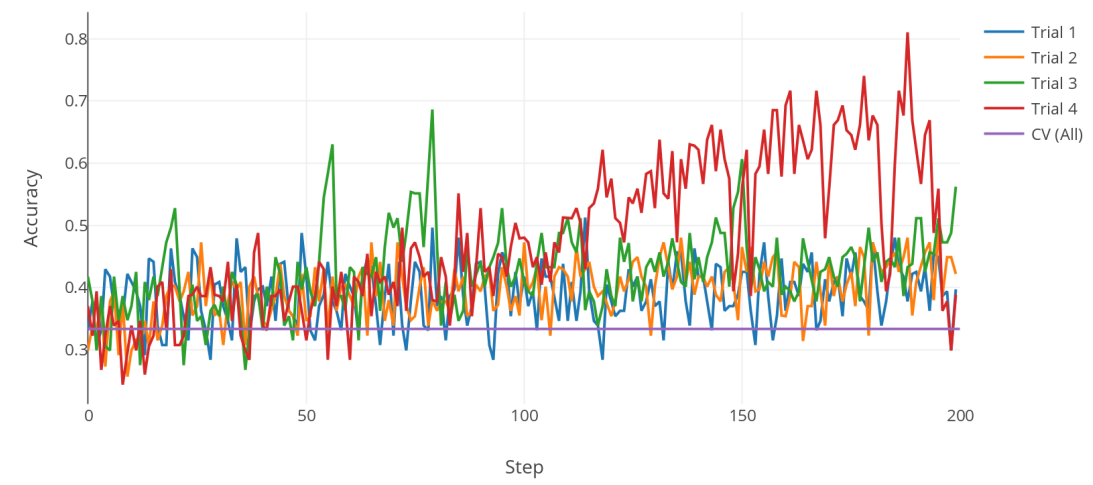

Figure 4.1: RNN-LSTM: Training classification accuracy for various Adam optimization strategy optimization parameters learning_rate, beta_1, beta_2, and epsilon.

These results can be from the RNN-LSTM's inability to identify any meaningful features after 40 epochs of the 633 training examples. The same results were seen when the model was trained for 200 epochs: The RNN-LSTM underfit the simulated data every time. Therefore, all model hyperparameters were increased. The resulting models successfully fit the input data and achieved significantly better accuracy when classifying the test set data. Further hyperparameter tuning showed that increasing the number of layers to be greater than 1 results in the model fitting the data appropriately. After 100 observations, Sigopt reported the RNN achieved 96.2\% accuracy. 
The CNN did not require much hyperparameter tuning. The CNN achieved near state-of-the-art success (accuracy > 95\%) on the first try. The CNN achieved 100\% accuracy after 15 optimization evaluations with SigOpt.

The final model hyperparameters were based on the first evaluation that classified the test set with $100 \%$ accuracy. These values are shown in Table 4.1. Similarly, the final performances are shown below in Figure 4.2.

Table 4.1: Final hyperparameters chosen for both models.

\begin{tabular}{l|c|c} 
Name & RNN-LSTM & CNN \\
\hline dropout_rate & 0.672 & 0.309 \\
\hline learning_rate & 0.00001 & 0.033 \\
\hline beta1 & 0.9 & 0.684 \\
\hline beta2 & 0.999 & 0.845 \\
\hline epsilon & $1 \mathrm{e}-08$ & 0.282 \\
\hline num_filt_1 & - & 16 \\
\hline kernel_size & - & 4 \\
\hline num_fc_1 & 31 & 6 \\
\hline n_layers & 4 & - \\
\hline n_hidden & 22 & -
\end{tabular}

\subsection{Final Results and Discussion}

Overall, both CNN and RNN models achieved above $90 \%$ accuracy on the validation and test dataset given sufficient time. Figure 4.2 depicts the accuracy curves during training across the training and validation datasets.

Different training parameters and hyperparameters were defined for each model to achieve these results. The training parameters of both models saw a change in the batch size batch_size and number of epochs $\mathrm{n}_{-}$epochs. The batch size was increased from 32 to 256 so each model update would better represent the dataset. The models 


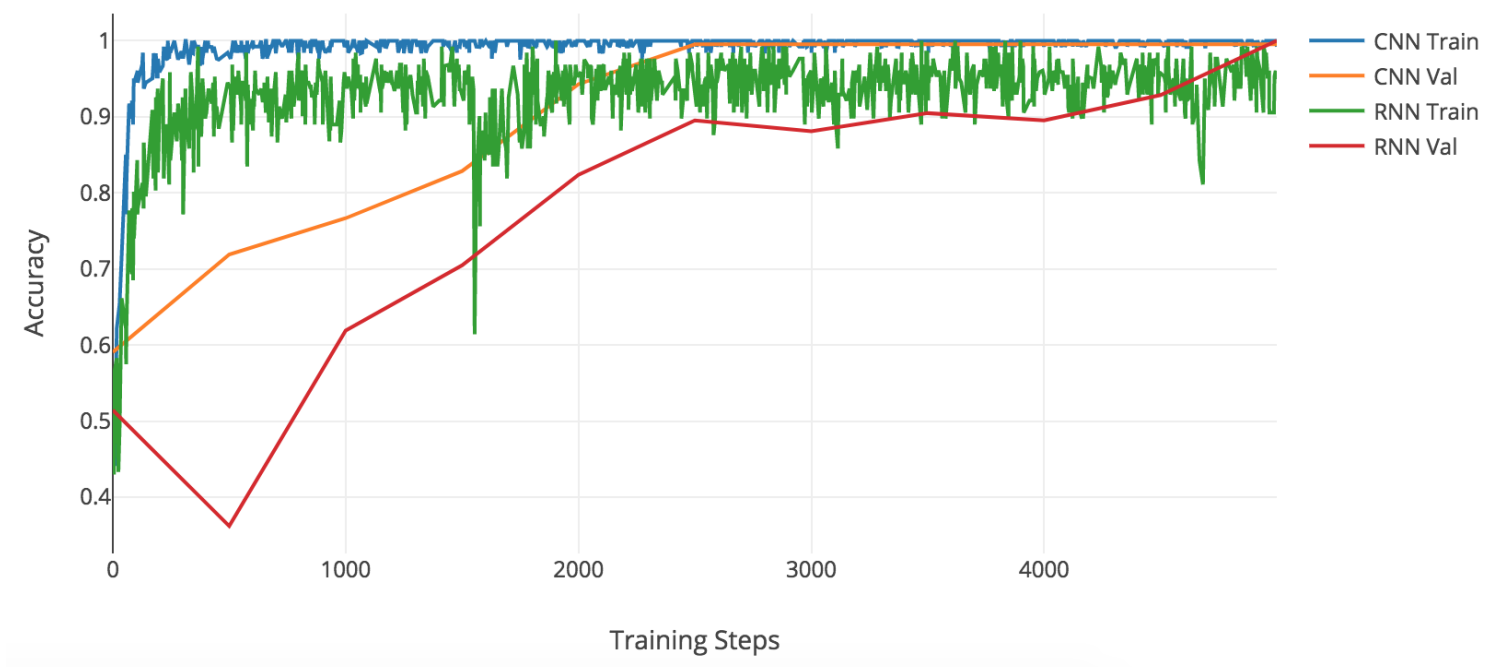

Figure 4.2: Classification Accuracy During Training

were ran until a validation dataset accuracy above $90 \%$ was observed, hence the final value $\mathrm{n} \_$epochs $=1000$.

The CNN requires significantly less time to train than the RNN-LSTM. This can be explained by looking at the mathematics behind the architectures. At each time step $t$, a RNN-LSTM must perform the following computations:

$$
\begin{aligned}
\mathbf{g}^{u} & =\sigma\left(\mathbf{W}^{u} \mathbf{h}_{t-1}+\mathbf{I}^{u} \mathbf{x}_{t}+\mathbf{b}_{\mathbf{u}}\right) \\
\mathbf{g}^{f} & =\sigma\left(\mathbf{W}^{f} \mathbf{h}_{t-1}+\mathbf{I}^{f} \mathbf{x}_{t}+\mathbf{b}_{\mathbf{f}}\right) \\
\mathbf{g}^{o} & =\sigma\left(\mathbf{W}^{o} \mathbf{h}_{t-1}+\mathbf{I}^{o} \mathbf{x}_{t}+\mathbf{b}_{\mathbf{o}}\right) \\
\mathbf{g}^{c} & =\tanh \left(\mathbf{W}^{c} \mathbf{h}_{t-1}+\mathbf{I}^{c} \mathbf{x}_{t}+\mathbf{b}_{\mathbf{c}}\right) \\
\mathbf{m}_{t} & =\mathbf{g}^{f} \odot \mathbf{m}_{t-1}+\mathbf{g}^{u} \odot \mathbf{g}^{c} \\
\mathbf{h}_{t} & =\tanh \left(\mathbf{g}^{o} \odot \mathbf{m}_{t}\right)
\end{aligned}
$$

where $\sigma$ is the logistic sigmoid function, $\odot$ represents elementwise multiplication, $\mathbf{W}^{u}, \mathbf{W}^{f}, \mathbf{W}^{o}, \mathbf{W}^{c}$ are recurrent weight matrices, $\mathbf{I}^{u}, \mathbf{I}^{f}, \mathbf{I}^{o}, \mathbf{I}^{c}$ are projection matrices, $\mathbf{b}$ is the bias vector, and $\mathbf{h}$ and $\mathbf{m}$ are hidden and memory vectors responsible for controlling state updates and outputs [18]. On the other hand, the input to some unit $x_{i}^{l}$ in layer $l$ is the sum of the previous layer's cells contributions $y$ multiplied by 
a filter $\omega$ with size $m[11]$. More clearly,

$$
x_{i}^{l}=\sum_{a=0}^{m} \omega_{a} y_{i+a}^{l-1}+b_{i}
$$

Compared directly against the fundamental equations behind a 1D convolution layer, one can see a stark contrast in complexity. Even if the filter or the number of previouslayer inputs are large in size, the CNN model is significantly simpler than the RNNLSTM model and thus is easier and faster to train.

The CNN also outperformed the RNN-LSTM model in classification capability. The RNN-LSTM model feeds the hidden layer from the previous layer from the previous step into the next step to provide information for tasks requiring long-range contextual information, but the input data here is based on short, simulated step responses. The additional computations aren't needed for classifying the data in this work; in fact, the RNN-LSTM is incorrectly biased on the built-up memory. The CNN is looking for specific patterns within windows of time within the time-series data. The clean, short simulated data does not vary in sequence length and has repeatable patterns within the data so the $\mathrm{CNN}$ is able to quickly train and accurately classify input data.

\subsection{Future Work}

This work laid down a foundation to explore an ANN-based TPMS, but much more work needs to be done before this technology can be applied. Future work should attempt to address the following aspects not covered here.

1. Improve the simulated data. In this work, all data was generated from a quarter-car model simulation. The simulation made many assumptions and is not representative of a real car model. A better simulation can be made by using a half-car or full-car model instead of a quarter-car model or making generally less assumptions.

2. Collect experimental data. Even better than simulated data is real exper- 
imental data. Collecting and analyzing real data can result in a better, more generalized classifier with no issues arising from training on simulated data. Furthermore, the data should be generalized away from a step function profile to the acceleration profile of general driving such that the TPMS can identify underpressurized tires at all times.

3. Develop the hardware. Instead of assuming the computational and electrical power required for the system exists, a more-thorough investigation should be performed to determine the validity of the claim. A theoretical system with the properly specified requirements would bring this work one step closer to reality.

4. Improve the algorithm. Further fine-tuning the training parameters and hyperparameters as well as adding and removing layers and features from the model architecture may result in more efficient and effective models.

\subsection{Conclusion}

Considering the various limitations of the work, these ANN-based TPMSs are far away from being applied across the automotive industry. Nonetheless, this work showed that both a CNN and RNN-LSTM model can be developed and trained on simulated training data to accurately classify unseen simulation data. This proves the algorithm's ability to identify unique patterns across each class and sort accordingly, all without any explicit instruction on the mechanical principles behind the data. With better data and appropriate hardware, vehicles may one day be equipped with ANN-based TPMS. 


\section{BIBLIOGRAPHY}

[1] A. Abdelghaffar, A. Hendy, O. Desouky, Y. Badr, S. Abdulla, and R. Tafreshi. Effects of Different Tire Pressures on Vibrational Transmissibility in Cars. In Mechanical Engineering and Mechatrnoics: 3rd International Conference, ICMEM 2014, Proceedings, 82014.

[2] C. Alippi, C. de Russis, and V. Piuri. A Neural-Network Based Control Solution to Air-Fuel Ratio Control for Automotive Fuel-Injection Systems. IEEE Transactions on Systems, Man, and Cybernetics, Part C, 33(2):259-268, July 2003. doi: 10.1109/TSMCC.2003.814035.

[3] Y. Bengio. Practical recommendations for gradient-based training of deep architectures. CoRR, abs/1206.5533, 2012.

[4] Y. Chen, T. Krishna, J. Emer, and V. Sze. Eyeriss: An Energy-Efficient Reconfigurable Accelerator for Deep Convolutional Neural Networks, Feb. 2016. doi: 10.4271/2002-01-1250.

[5] A. Deshpande. A Beginner's Guide to Understanding Convolutional Neural Networks, 82015.

[6] I. Dewancker, M. McCourt, S. Clark, P. Hayes, A. Johnson, and G. Ke. A Stratified Analysis of Bayesian Optimization Methods. CoRR, abs/1603.09441, 2016.

[7] J. Dixon. The Shock Absorber Handbook. Wiley-PEPublishing Series. John Wiley \& Sons, 2007.

[8] G. Dorffner. Neural Networks for Time Series Processing. Neural Network World, 6:447-468, 1996.

[9] J. C. B. Gamboa. Deep learning for time-series analysis. CoRR, abs/1701.01887, 2017.

[10] M. Giaraffa. Springs \& Dampers, Part Three, 2017. 
[11] A. Gibiansky. Math [Code], Feb 2014.

[12] K. Habib. PE 16-007. Technical report, NHTSA, Jan. 2017.

[13] M. Hollemans. Convolutional Neural Networks on the iPhone with VGGnet, 8 2016.

[14] D. V. III and D. Vomhof. Individual vehicle data search service. Technical report, 4N6XPRT Systems, La Mesa CA 91942, 52012.

[15] S. Ioffe and C. Szegedy. Batch normalization: Accelerating deep network training by reducing internal covariate shift. CoRR, abs/1502.03167, 2015.

[16] R. Jazar. Vehicle Dynamics: Theory and Application. Springer-Verlag New York, 2 edition, 2014.

[17] T. Jiang, S. Petrovic, U. Ayyer, A. Tolani, and S. Husain. Self-Driving Cars: Disruptive or Incremental? Applied Innovation Review, 4(1):3-22, June 2015.

[18] F. Karim, S. Majumdar, H. Darabi, and S. Chen. LSTM Fully Convolutional Networks for Time Series Classification. CoRR, abs/1709.05206, 2017.

[19] U. Karn. An intuitive explanation of convolutional neural networks, 82016.

[20] D. P. Kingma and J. Ba. Adam: A Method for Stochastic Optimization. CoRR, abs/1412.6980, 2014.

[21] A. Kubba and K. Jiang. A Comprehensive Study on Technologies of Tyre Monitoring Systems and Possible Energy Solutions. Sensors, 14(6):10306-10345, June 2014. doi: 10.3390/s140610306.

[22] Z. C. Lipton. A critical review of recurrent neural networks for sequence learning. CoRR, abs/1506.00019, 2015.

[23] MathWorks. Automotive Suspension, May 2016.

[24] J. Mccaffrey. Why You Should Use Cross-Entropy Error Instead of Classification Error or Mean Squared Error for Neural Network Classifier Training, 112013. 
[25] NHTSA. Docket No. NHTSA 2205-20586, 2005.

[26] NHTSA. Tire Pressure Maintenance - A Statistical Investigation, Apr. 2009.

[27] NHTSA. IV. Tire Pressure Monitoring Systems, 2016.

[28] NHTSA. Tires, 2016.

[29] C. Olah. Understanding LSTM Networks, 82015.

[30] J. Overton, B. Mills, and C. Ashley. The Vertical Response Characteristics of the Non-Rolling Tyre. In Proceedings of The Institution of Mechanical Engineers, Automobile Division 1947-1970, volume 184, pages 25-40, 6 1969.

[31] N. Persson, F. Gustafsson, and M. Drevo. Indirect Tire Pressure Monitoring Using Sensor Fusion. In SAE Technical Paper", publisher $=S A E$ International, journal $=S A E$, year $=2002$, month $=$ Mar, note $=$ doi: 10.4271/2002-01-1250, url = https://doi.org/10.4271/2002-01-1250, doi $=$ 10.4271/2002-01-1250.

[32] E. Phaisangittisagul. An analysis of the regularization between 12 and dropout in single hidden layer neural network. In 2016 7th International Conference on Intelligent Systems, Modelling and Simulation (ISMS), pages 174-179, 1 2016.

[33] A. Plieninger. Deep Learning Neural Networks on Mobile Platforms. Master's thesis, Technische Universitat Munchen, Oct. 2015.

[34] P. Ramachandran, B. Zoph, and Q. V. Le. Searching for Activation Functions. CoRR, abs/1710.05941, 2017.

[35] D. Silver and D. Hassabis. Alphago: Mastering the ancient game of Go with Machine Learning, 12016.

[36] R. Tayler, L. Bashford, and M. Shcrock. Methods for Measuring Vertical Tire Stiffness. Transactions of the ASAE, 43(6):1415-1419, 2000. doi: 10.13031/2013.3039. 
[37] TireWise. Tire Maintenance, 2016.

[38] United States Senate and House of Representatives. Transportation Recall Enhancement, Accountability, and Documentation (TREAD) Act, Nov. 2000.

[39] V. Wiggins, S. Engquist, and L. Looper. Neural Network Applications: A Literature Review. Technical report, Air Force, Nov. 1992.

[40] Y. Zheng, Q. Liu, E. Chen, Y. Ge, and J. Zhao. Time Series Classification Using Multi-Channels Deep Convolutional Neural Networks. In Web-Age Information Management: 15th International Conference, WAIM 2014, Proceedings, pages 298-310, 62014. 


\title{
APPENDICES
}

\section{Appendix A \\ DERIVATIONS OF SIMULATION INPUT PARAMETERS}

\begin{abstract}
All constants used as simulation input variables are derived as follows. Except for identifying $m_{u}$, all of these calculations are performed in CalculateTireStiffness.m and

CalculateSuspensionStiffnessDamping.m.
\end{abstract}

\section{A.1 $m_{s}$}

$m_{s}$ is simply taken from [14] and divided by 4 to account for the quarter-car model.

$$
m_{s}=1109 \mathrm{~kg} / 4=277.25 \mathrm{~kg}
$$

\section{A.2 $k_{s}$}

Assuming that the tire in use across all vehicles is a radial-ply $165 \times 13$ tire (a very common tire size found on most passenger vehicles), a linear model for static stiffness based on tire inflation pressure can be used [30]. The model is graphically presented in Figure A.1 and expressed by equation A.2. The model is only accurate above 15 psi-an acceptable limitation as 15 psi is well below the threshold for "underpressurized."

$$
k_{s}=30.185 p_{u}+46.375
$$




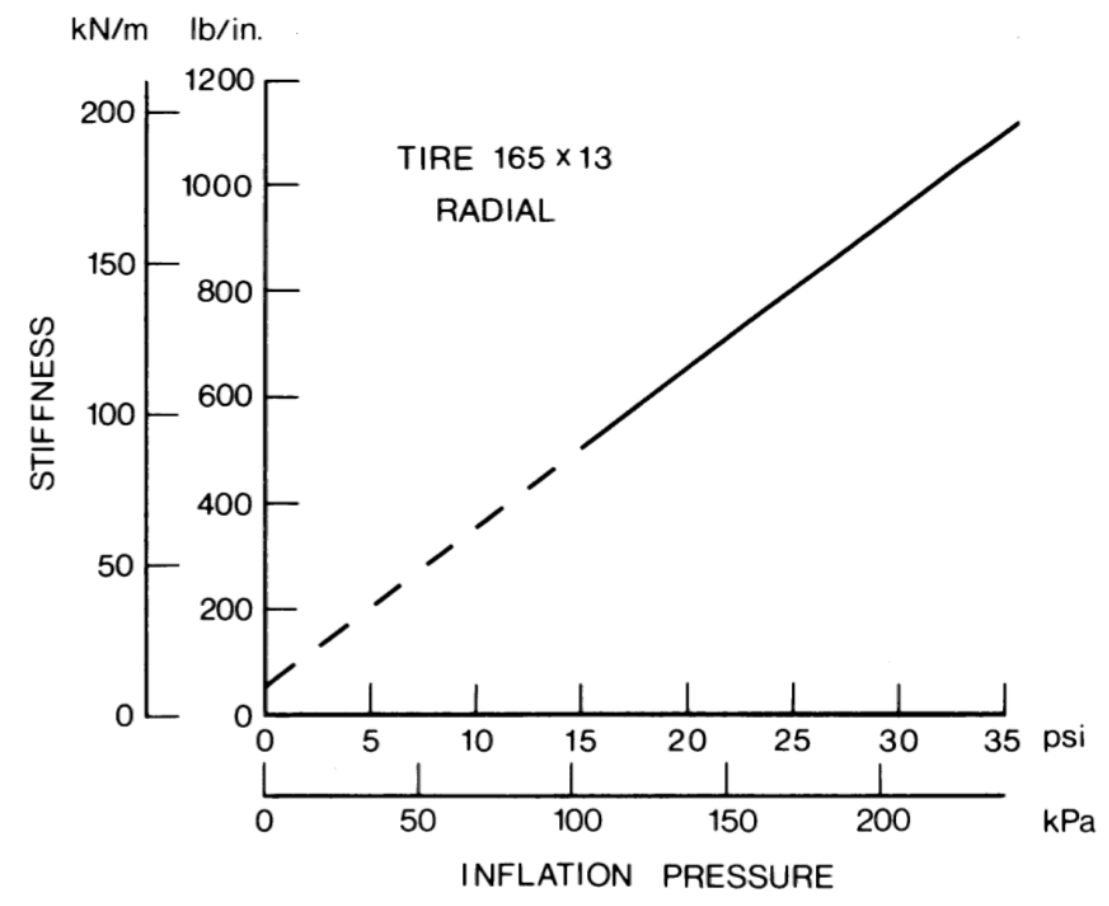

Figure A.1: Static stiffness vs. inflation pressure for a radial-ply car tire. [30]

\section{A.3 $m_{u}$}

Utilizing the average quarter-car ratio of the sprung to unsprung masses and the one can identify the expected value for $m_{u}[16]$ :

$$
\begin{aligned}
\varepsilon=\frac{m_{s}}{m_{u}}=8 \Rightarrow m_{u} & =\frac{m_{s}}{\varepsilon} \\
& =\frac{277.25 \mathrm{~kg}}{8} \\
& =34.69 \mathrm{~kg}
\end{aligned}
$$

It should be noted that $m_{u}$ should vary with tire pressure due to the additional air inside the tires. However, the mass of the air is insignificant relative to the rest of the unsprung mass $((<0.1 \%)$. Nonetheless, the mass of the air is calculated and included in the unsprung mass for these simulations. The calculations are performed in CalculateTireWeight.m. 


\section{A.4 $k_{s}$ and $c_{s}$}

To identify the suspension's stiffness and damping coefficients, assume that the suspension is tuned for a properly-inflated tire. With $p_{u}=32$ psi, equations A.2 and A.3 give the $k_{u}=6979.53 \mathrm{kPa}$ and $m_{u}=34.69 \mathrm{~kg}$ respectively. With these values, one can find the natural frequency of the unsprung mass $\omega_{u}$ :

$$
\begin{aligned}
\omega_{u} & =\sqrt{\frac{k_{u}}{m_{u}}} \\
& =\sqrt{\frac{6979.53 \mathrm{kPa}}{34.69 \mathrm{~kg}}} \\
& =448.612 \mathrm{~Hz}
\end{aligned}
$$

The average quarter-car ratio for sprung and unsprung natural frequences is used to identify the sprung mass's natural frequency $\omega_{s}$.

$$
\begin{aligned}
\alpha=\frac{\omega_{s}}{\omega_{u}}=0.1 \Rightarrow \omega_{s} & =\alpha \omega_{u} \\
& =(0.1)(448.61 \mathrm{~Hz}) \\
& =44.86 \mathrm{~Hz}
\end{aligned}
$$

We already know that $m_{s}=277.25 \mathrm{~kg}$, so identifying $k_{s}$ is trivial.

$$
\begin{aligned}
\omega_{s}=\sqrt{\frac{k_{s}}{m_{s}}} \Rightarrow k_{s} & =\omega_{s}^{2} m_{s} \\
& =(44.86 \mathrm{~Hz})^{2}(277.25 \mathrm{~kg}) \\
& =557.97 \mathrm{kPa}
\end{aligned}
$$

To calculate $c_{s}$, we can use the relationship between $\omega_{s}$ and the damping ratio $\zeta=\frac{c_{s}}{c}$, where $c$ is the critical damping coefficient. Numerous sources suggest the proper damping ratio in passenger vehicles to be between 0.2 and 0.3 [7] [10]. For this work, we define $\zeta=0.25$. 


$$
\begin{aligned}
\zeta=\frac{c_{s}}{2 m_{s} \omega_{s}} \Rightarrow c_{s} & =2 \zeta m_{s} \omega_{s} \\
& =2(0.25)(277.25 \mathrm{~kg})(44.86 \mathrm{~Hz}) \\
& =6218.8 \mathrm{~Pa}-\mathrm{s}
\end{aligned}
$$




\section{Appendix B}

\section{SOURCE CODE}

\section{B.1 cnn_model.py}

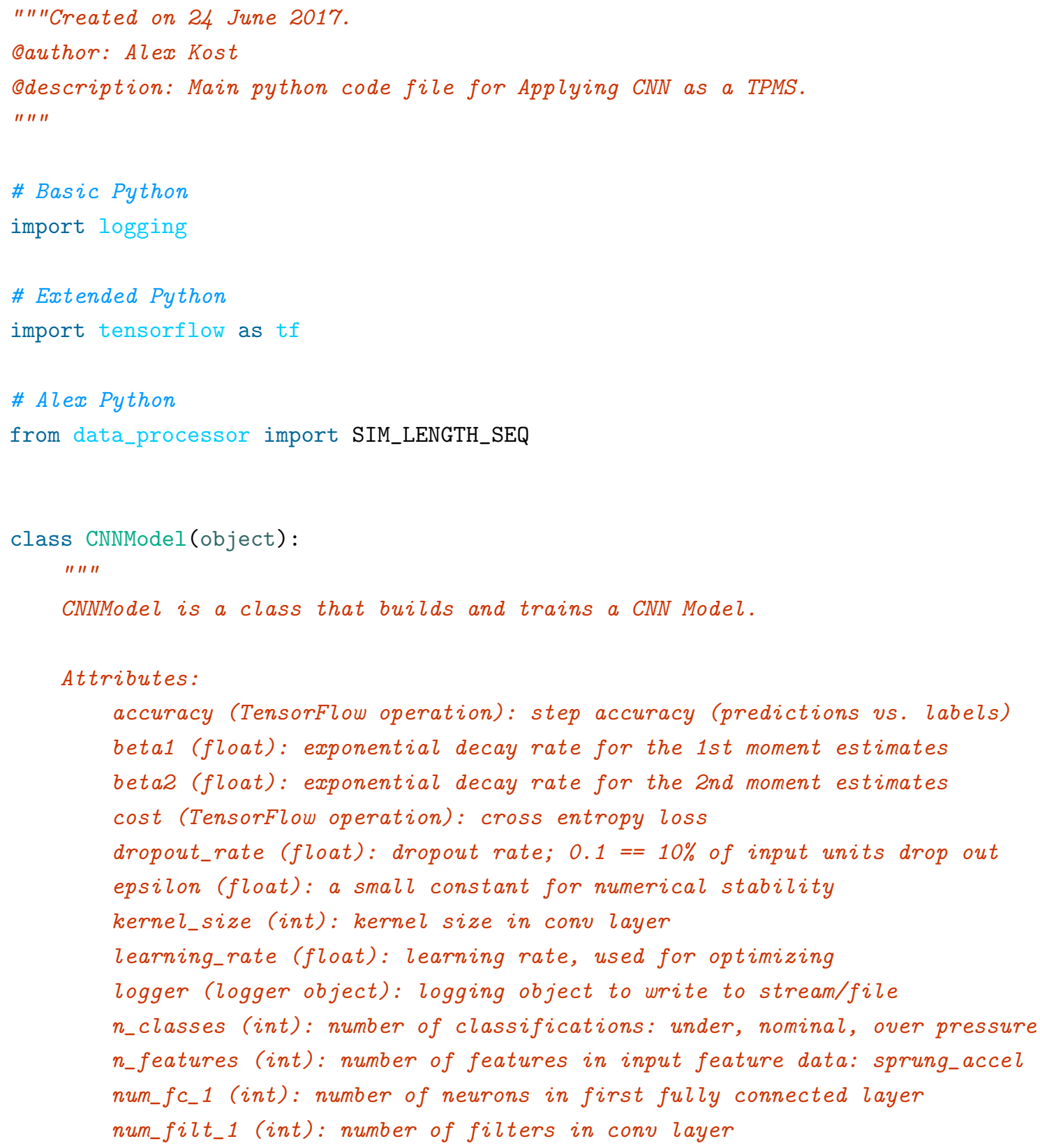


optimizer (Tensorflow operation): AdamOptimizer operation used to train

$\hookrightarrow \quad$ the model

summary_op (Tensorflow operation): summary operation of all tf.summary $\hookrightarrow$ objects

\section{$\hookrightarrow$ training/evaluating}

$x$ (Tensorflow placeholder): input feature data

$y$ (Tensorflow placeholder): input label data " " "

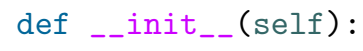


self.summary_op = None

$\hookrightarrow \quad \operatorname{data}$

def build_model(self):

""Build the CNN Model.""

input_shape $=$ [None, SIM_LENGTH_SEQ, self.n_features] if self.n_features > $\hookrightarrow \quad 1$ else [None, SIM_LENGTH_SEQ]

self.x = tf.placeholder(tf.float32, shape=input_shape, name='input_data')

self.y = tf.placeholder(tf.int64, shape=[None], name='input_labels')

with tf.variable_scope("Reshape_Data"):

\# tf.nn.conv2d requires inputs to be shaped as follows:

\# [batch, in_height, in_width, in_channels]

\# so -1 = batch size, should adapt accordingly

\# in_height = "height" of the image (so one dimension)

\# in_width = "width" of image

x_reshaped $=\mathrm{tf} . \mathrm{reshape}(\operatorname{self} . \mathrm{x}$, [-1, SIM_LENGTH_SEQ, self.n_features])

self.logger.debug('Input dims: \{\} '.format (x_reshaped.get_shape()))

with tf.variable_scope("ConvBatch1") :

$x_{\text {_bn }}=\mathrm{tf}$. contrib.layers.batch_norm(inputs $=\mathrm{x}_{-}$reshaped, is_training=self .trainable, updates_collections=None)

conv1 = tf.layers. $\operatorname{conv1d}\left(\right.$ inputs $=x_{-} b n$, filters=self.num_filt_1, kernel_size=[self.kernel_size])

self.logger.debug('Conv1 output dims: \{\} '.format(conv1.get_shape()))

with tf.variable_scope("Fully_Connected1"):

conv2_flatten $=$ tf.layers.flatten $(\operatorname{conv1}$, name='Flatten' $)$

$f c 1=$ tf.contrib.layers.fully_connected (inputs=conv2_flatten, num_outputs=self.num_fc_1,

$\hookrightarrow \quad$ weights_initializer=tf . contrib.layers. $x a$

$\hookrightarrow \quad$ biases_initializer=tf . constant_initializ

$\hookrightarrow \quad$ normalizer_fn=tf.contrib.layers.batch_nc

$\hookrightarrow$ normalizer_params $=\{$ 'is_training ' :

$\hookrightarrow$ self.trainable,

$\hookrightarrow \quad$ 'updates_collections '

$\hookrightarrow \quad$ None\}) 
$f c 1=t f$. layers.dropout (inputs=fc1, rate=self.dropout_rate, $\hookrightarrow$ training=self .trainable) self.logger.debug('FCon1 output dims: \{\} '.format(fc1.get_shape()))

with tf.variable_scope("Fully_Connected2"): pred $=$ tf . contrib.layers.fully_connected (inputs $=f c 1$, num_outputs=self.n_classes,

$\hookrightarrow \quad$ weights_initializer=tf .contrib.layers.x

self.logger.debug('FCon2 output dims: \{\} '.format(pred.get_shape())) tf.summary.histogram('pred', pred)

\# MEASURE MODEL ERROR

\# Cross-Entropy: "measuring how inefficient our predictions are for

$\hookrightarrow$ describing the truth"

\# http://colah.github.io/posts/2015-09-Visual-Information/

\#

$\hookrightarrow$ https://stackoverflow.com/questions/41689451/valueerror-no-gradients-provided-for-ar

\# Use sparse softmax because we have mutually exclusive classes

\# logits must be [batch_size, num_classes], label must be [batch_size]

\# $t f$.reduce_mean = reduces tensor to mean scalar value of tensor

with tf.variable_scope("Softmax"):

cross_entropy $=$

$\hookrightarrow$ tf.nn.sparse_softmax_cross_entropy_with_logits(logits=pred,

$\hookrightarrow \quad$ labels=self $\cdot \mathrm{y})$

self.cost $=t f . r e d u c e \_m e a n\left(c r o s s \_e n t r o p y\right.$, name $\left.=^{\prime} \operatorname{cost}^{\prime}\right)$

tf.summary.scalar('cross_entropy_loss', self.cost)

\# EVALUATE OUR MODEL

\# tf.argmax $=$ returns index of the highest entry in a tensor along some $\hookrightarrow \quad$ axis.

\# Predictions are probabilities corresponding to class (ex. [0.7 0.2 $\hookrightarrow \quad 0.1]$ )

\# tf.argmax returns the most probable label (ex. 0)

\# tf.equal = compares prediction to truth, returns list of bools ( $T$ if $\hookrightarrow$ correct, Fif not)

\# tf.reduce_mean = reduces tensor to mean scalar value of tensor

\# tf. cast $=$ convert bools to 1 and 0

with tf.variable_scope("Evaluating"):

correct_pred $=$ tf.equal $(t f . \operatorname{argmax}($ pred, 1$)$, self.y)

self.accuracy $=t f . r e d u c e \_m e a n\left(t f . c a s t\left(c o r r e c t \_p r e d, t f . f l o a t 32\right)\right.$ )

tf.summary.scalar('accuracy', self.accuracy) 
\# OPTIMIZE OUR MODEL

with tf.variable_scope("Optimizing"):

self.optimizer $=$ tf.train.AdamOptimizer(self.learning_rate,

beta1=self . beta1,

beta2 $=$ self. beta2,

141

$\hookrightarrow \quad$ epsilon=self.epsilon).minimize(self.cost 


\section{B.2 rnn model.py}

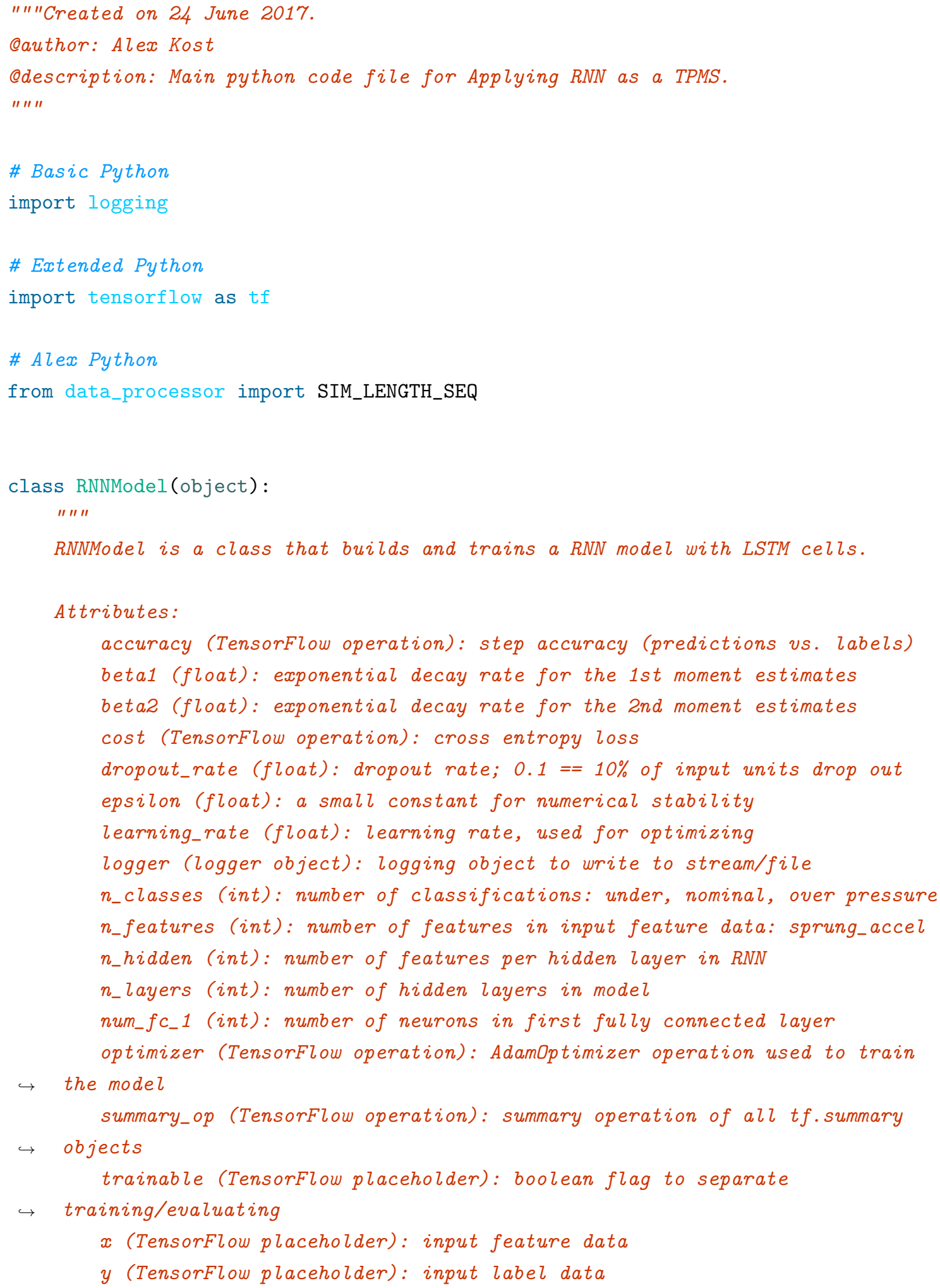




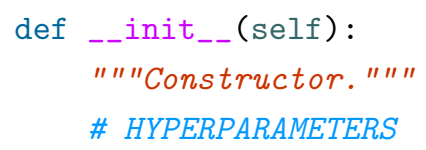


107

self.y = tf.placeholder(tf.int64, shape=[None], name='input_labels')

if input_shape $==$ [None, SIM_LENGTH_SEQ] : with tf.variable_scope("Reshape_Data"):

\# tf.nn. conv2d requires inputs to be shaped as follows:

\# [batch_size, max_time, ...]

\# so -1 = batch size, should adapt accordingly

\# max_time = SIM_LENGTH_SEQ

\# ... = self. $n_{-} f e a t u r e s$

x_reshaped $=$ tf.reshape $(\operatorname{self} . \mathrm{x},[-1$, SIM_LENGTH_SEQ, $\hookrightarrow$ self.n_features])

self.logger.debug ('Input dims: \{\} '.format (x_reshaped.get_shape()))

with tf.variable_scope("LSTM_RNN") :

\# add stacked layers if more than one layer

if self.n_layers > 1 :

cell = tf.contrib.rnn.MultiRNNCell ([self._setup_lstm_cell() for _ $\hookrightarrow \quad$ in range(self.n_layers)],

else:

cell = self._setup_lstm_cell ()

\# outputs $=$ [batch_size, max_time, cell.output_size]

\# outputs contains the output of the last layer for each time-step

outputs, _ = tf.nn.dynamic_rnn(cell=cell,

inputs $=x \_r e s h a p e d$,

dtype $=$ tf. float32)

self.logger.debug('dynamic_rnn output dims:

$\hookrightarrow\{\}^{\prime}$.format (outputs.get_shape()))

\# We transpose the output to switch batch size with sequence size -

$\hookrightarrow$ http://monik.in/a-noobs-guide-to-implementing-rnn-lstm-using-tensorflow/

outputs = tf.transpose (outputs, $[1,0,2]) \quad$ \# Now shape =

$\hookrightarrow \quad$ [max_time, batch_size, cell.output_size]

last = outputs[-1] \#Last slice is of

$\hookrightarrow$ shape [batch_size, cell.output_size]

self.logger.debug('last output dims: \{\} '.format(last.get_shape()))

with tf.variable_scope("Fully_Connected1"):

$f c 1=$ tf.contrib.layers.fully_connected(inputs=last, num_outputs=self.num_fc_1,

$\hookrightarrow \quad$ weights_initializer=tf . contrib.layers.xa 
$\hookrightarrow \quad$ 'updates_collections ' $\hookrightarrow$ None\})




\# Predictions are probabilities corresponding to class (ex. [0.7 0.2 $\hookrightarrow \quad 0.1])$

\# tf.argmax returns the most probable label (ex. 0)

\# tf.equal = compares prediction to truth, returns list of bools ( $T$ if $\hookrightarrow$ correct, $F$ if not)

\# $t f . r e d u c e \_m e a n=$ reduces tensor to mean scalar value of tensor

\# tf. cast $=$ convert bools to 1 and 0

with tf.variable_scope ("Evaluating"):

correct_pred = tf.equal (tf.argmax $(\operatorname{pred}, 1)$, self.y)

self.accuracy $=$ tf.reduce_mean(tf.cast (correct_pred, tf.float32))

tf.summary.scalar ('accuracy', self.accuracy)

\# OPTIMIZE OUR MODEL

with tf.variable_scope ("Optimizing") :

self.optimizer = tf.train.Adam0ptimizer(self.learning_rate, beta1 $=$ self . beta1, beta2 $=$ self. beta 2 ,

$\hookrightarrow \quad$ epsilon=self.epsilon).minimize(self.cost

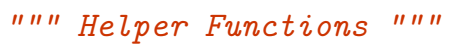




\section{B.3 data_processor.py}

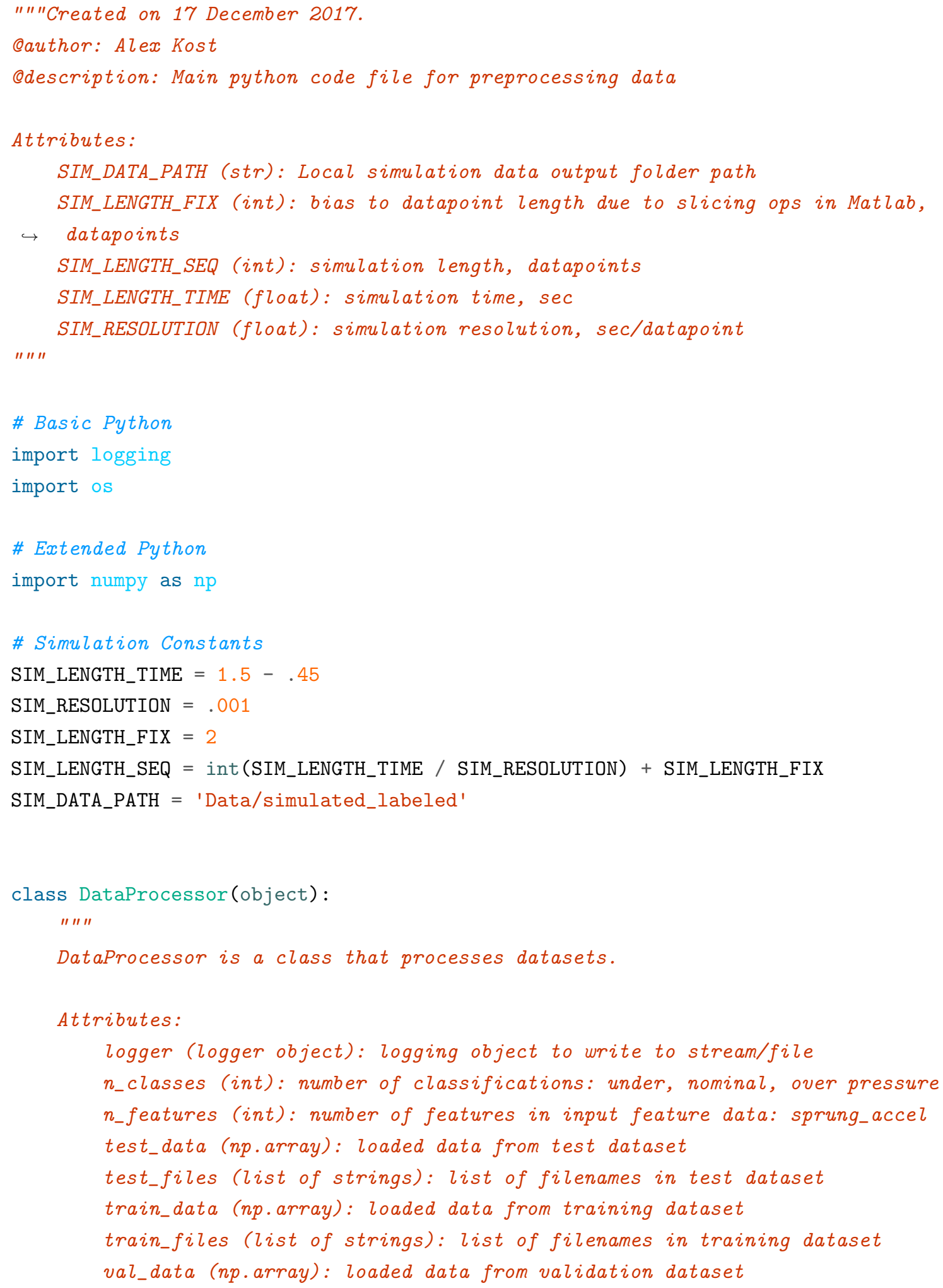




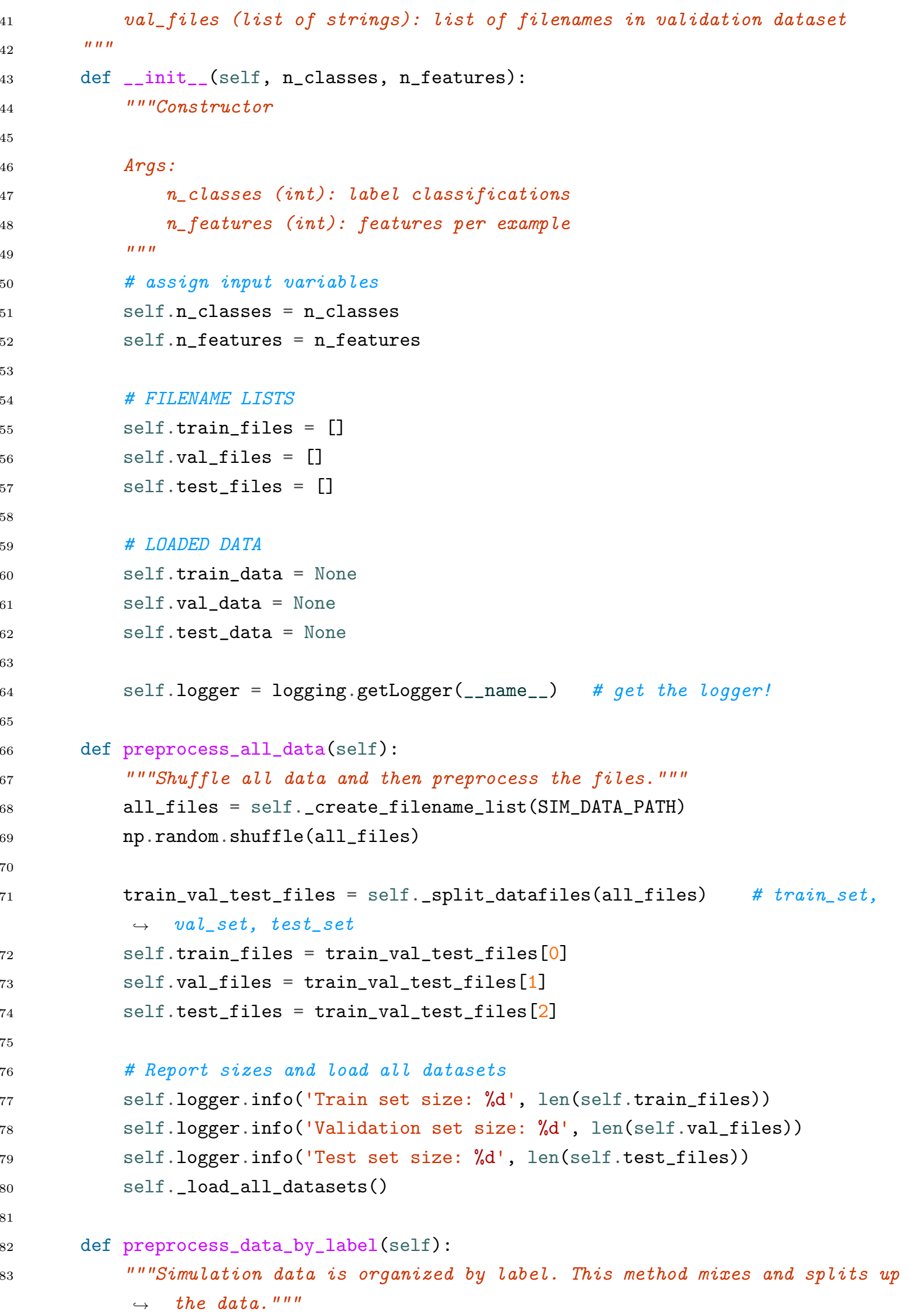


for $i$ in range(self.n_classes):

modified_data_path = os.path.join(SIM_DATA_PATH, str(i))

class_files $=$ self._create_filename_list(modified_data_path)

\# get files for each thing

result $=$ self._split_datafiles(class_files) \# train_set, val_set, $\hookrightarrow$ test_set

self.train_files.extend (result [0])

self.val_files.extend (result [1])

self.test_files.extend (result [2])

self.logger.debug $(1 \% \mathrm{~d} / \% \mathrm{~d} / \% \mathrm{~d}$ added to train/val/test set from class $\hookrightarrow \%$ d.,

$$
\begin{aligned}
& \operatorname{len}(\operatorname{result}[0]), \operatorname{len}(\operatorname{result}[1]), \\
& \operatorname{len}(\operatorname{result}[2]), i)
\end{aligned}
$$

\# Shuffle data

np.random.shuffle(self.train_files)

np.random.shuffle(self.val_files)

np.random.shuffle(self.test_files)

\# Report sizes and load all datasets

self.logger.info('Train set size: \%d', len(self.train_files))

self.logger.info('Validation set size: $\% d$ ', len(self.val_files))

self.logger.info('Test set size: \%d', len(self.test_files))

self._load_all_datasets()

"" Helper Functions "" "

def _load_all_datasets(self):

"" "Assign class member variables after processing filenames." ""

self.train_data = self._load_data(self.train_files) \#features, labels

self.val_data $=$ self._load_data(self.val_files) \# features,

$\rightarrow$ labels

self.test_data $=$ self._load_data(self.test_files) \# features,

$\rightarrow$ labels

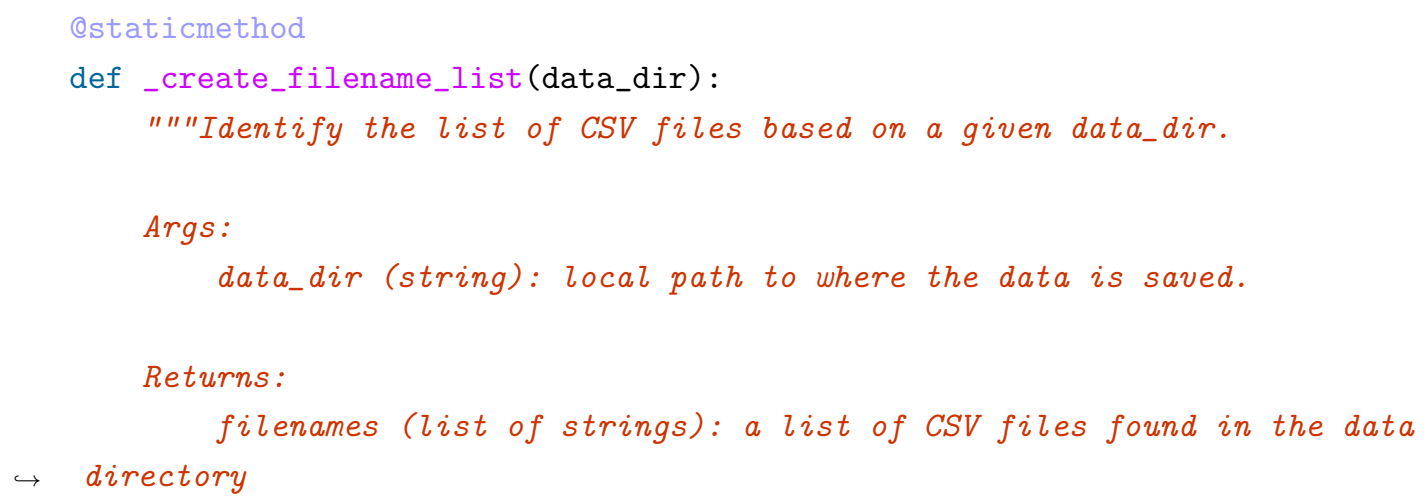









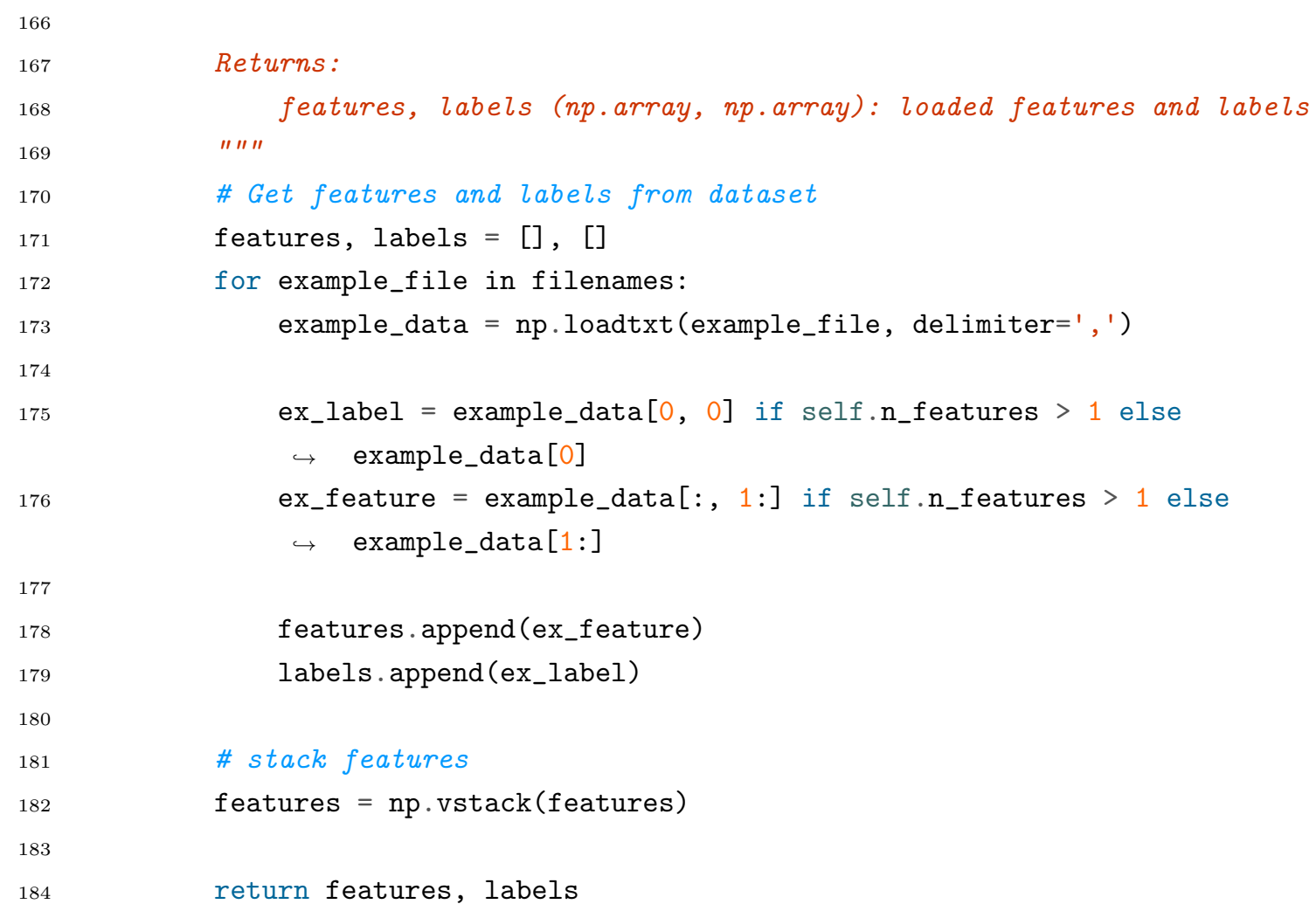




\section{B.4 train.py}

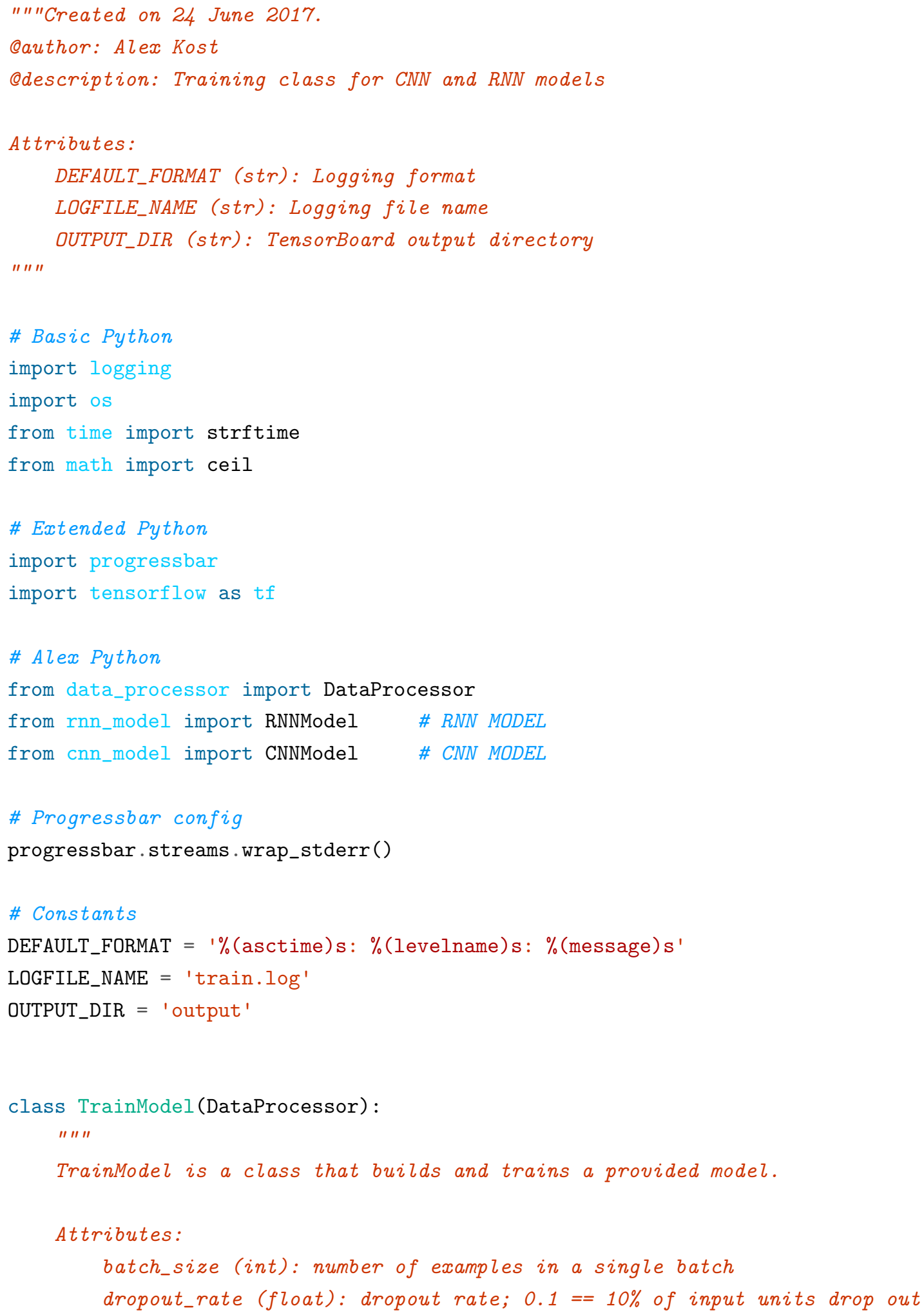




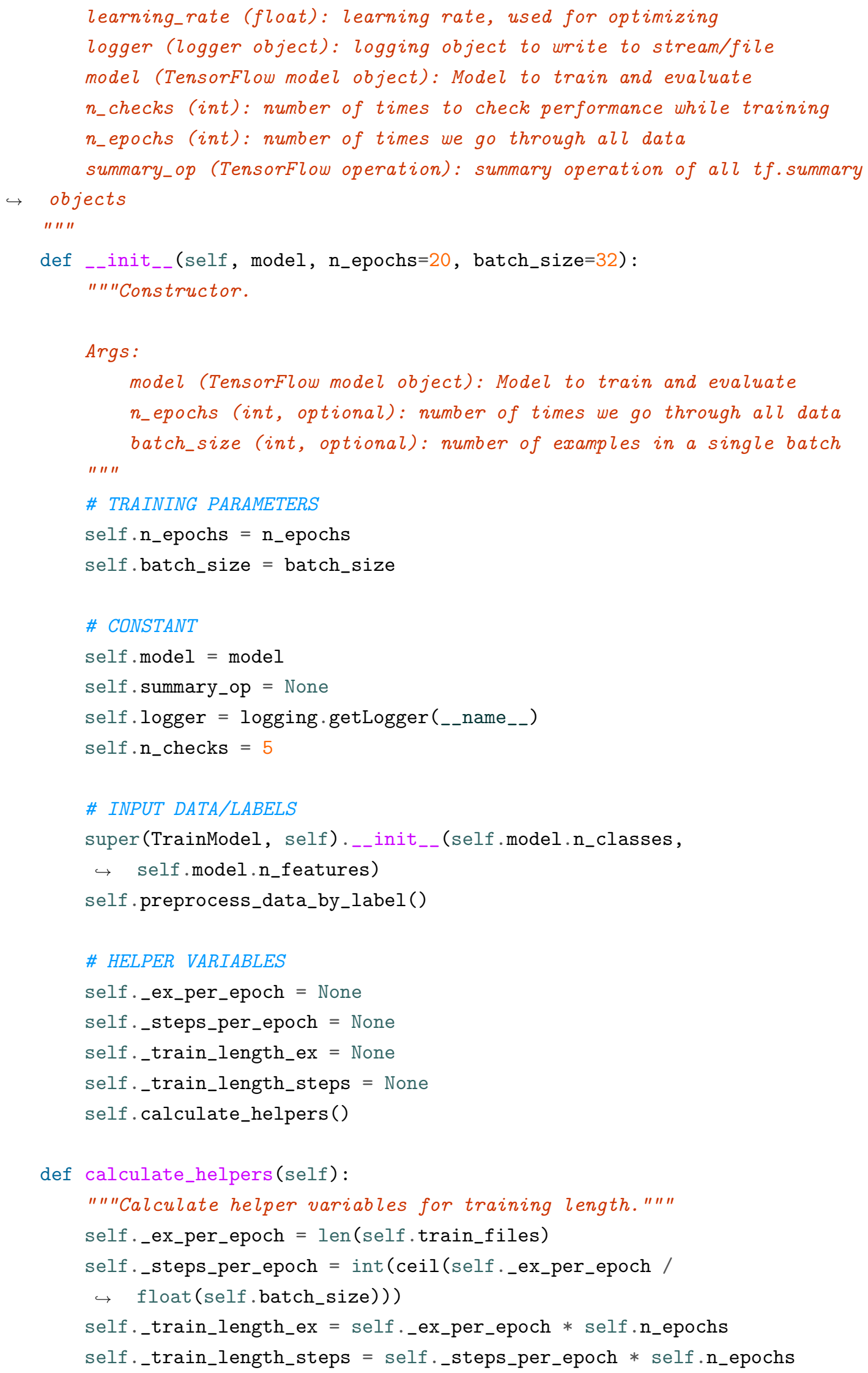


self.logger.debug('self._ex_per_epoch: \%d', self._ex_per_epoch) self.logger.debug('self._steps_per_epoch: \%d', self._steps_per_epoch) self.logger.debug('self._train_length_ex: \%d', self._train_length_ex) self.logger.debug('self._train_length_steps: \%d', $\hookrightarrow$ self._train_length_steps)

def train_model(self, use_tensorboard=True): "" "Train the model.

Args:

use_tensorboard (bool, optional): Description

Returns:

TYPE: Description " " " 


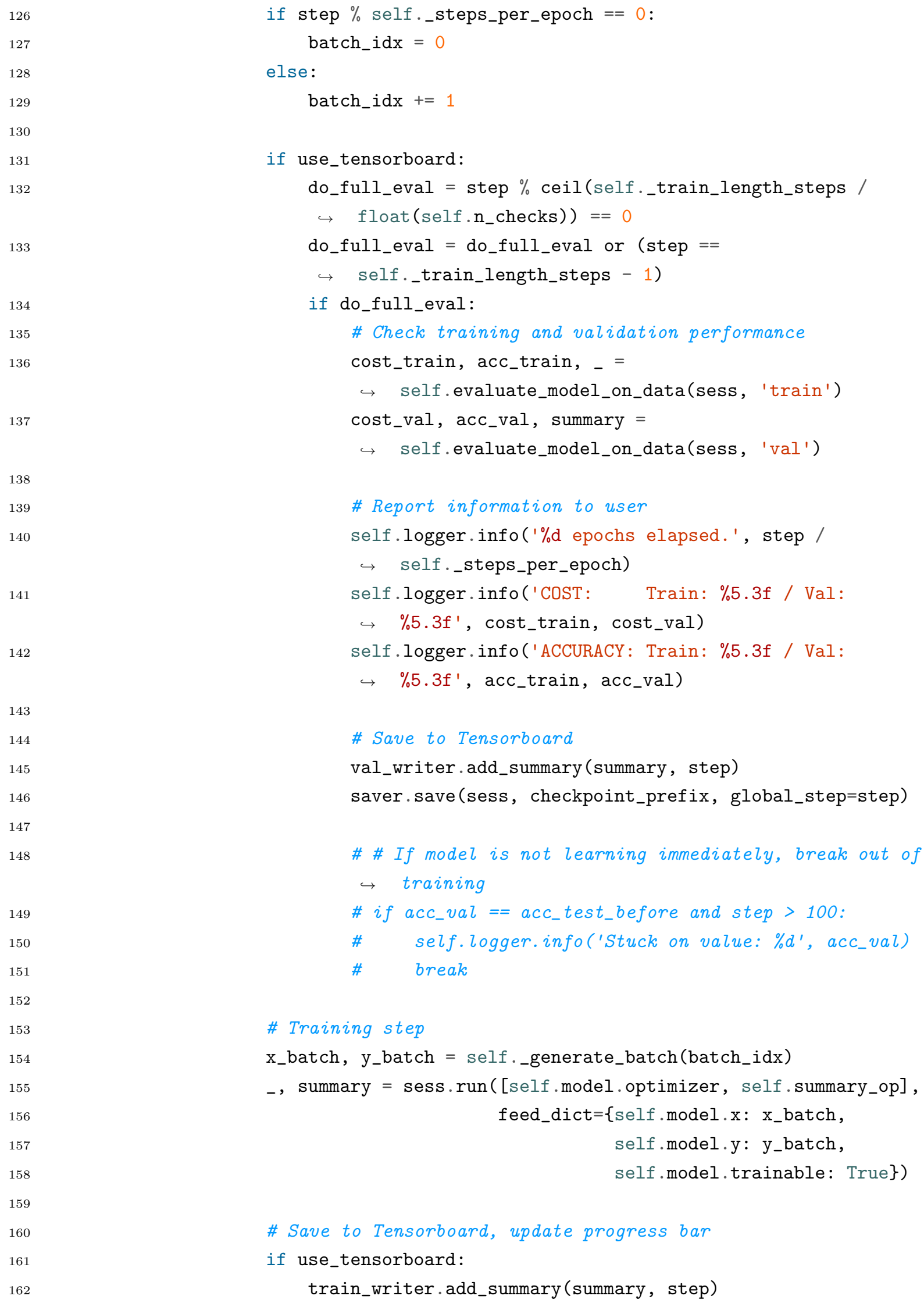




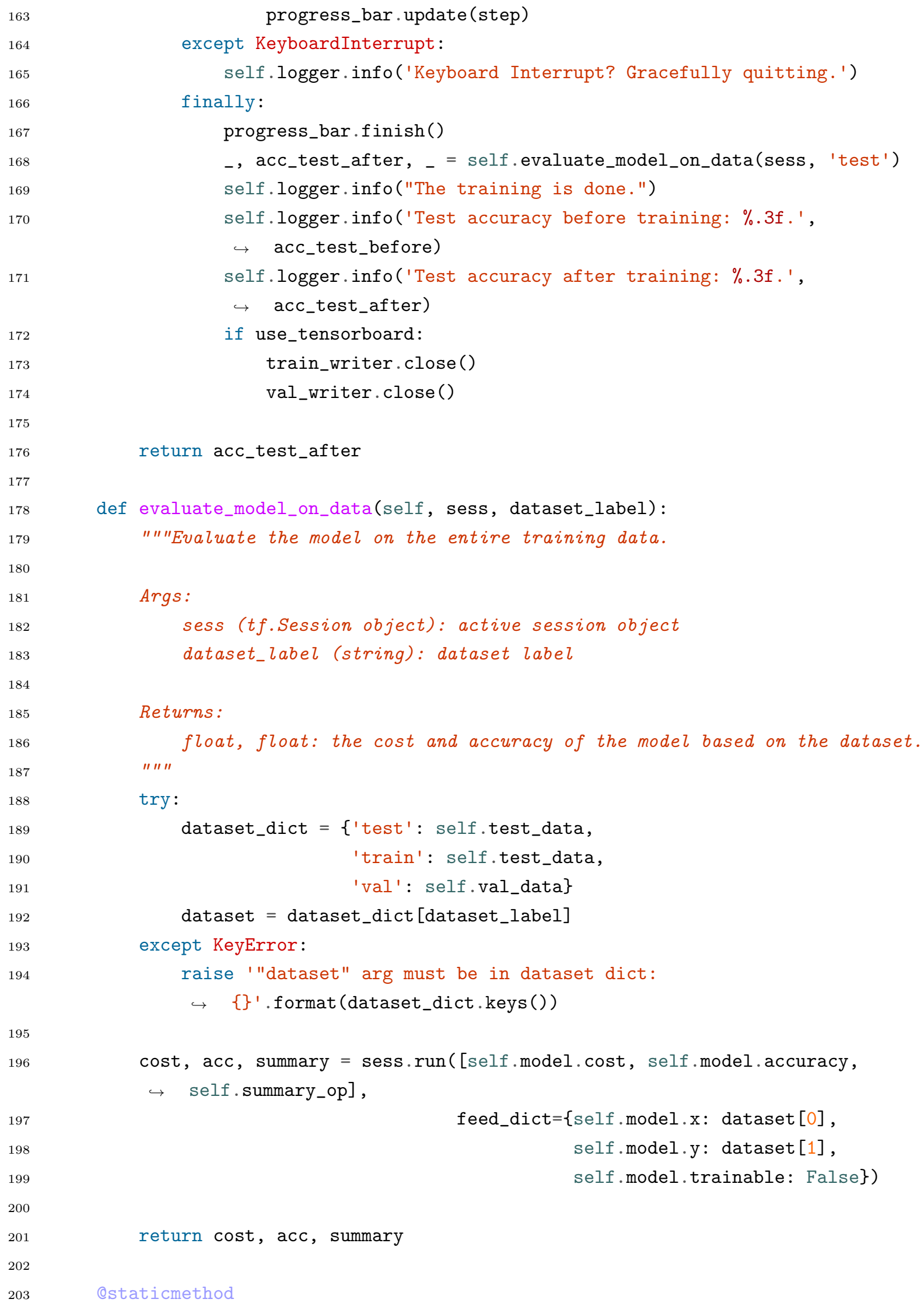




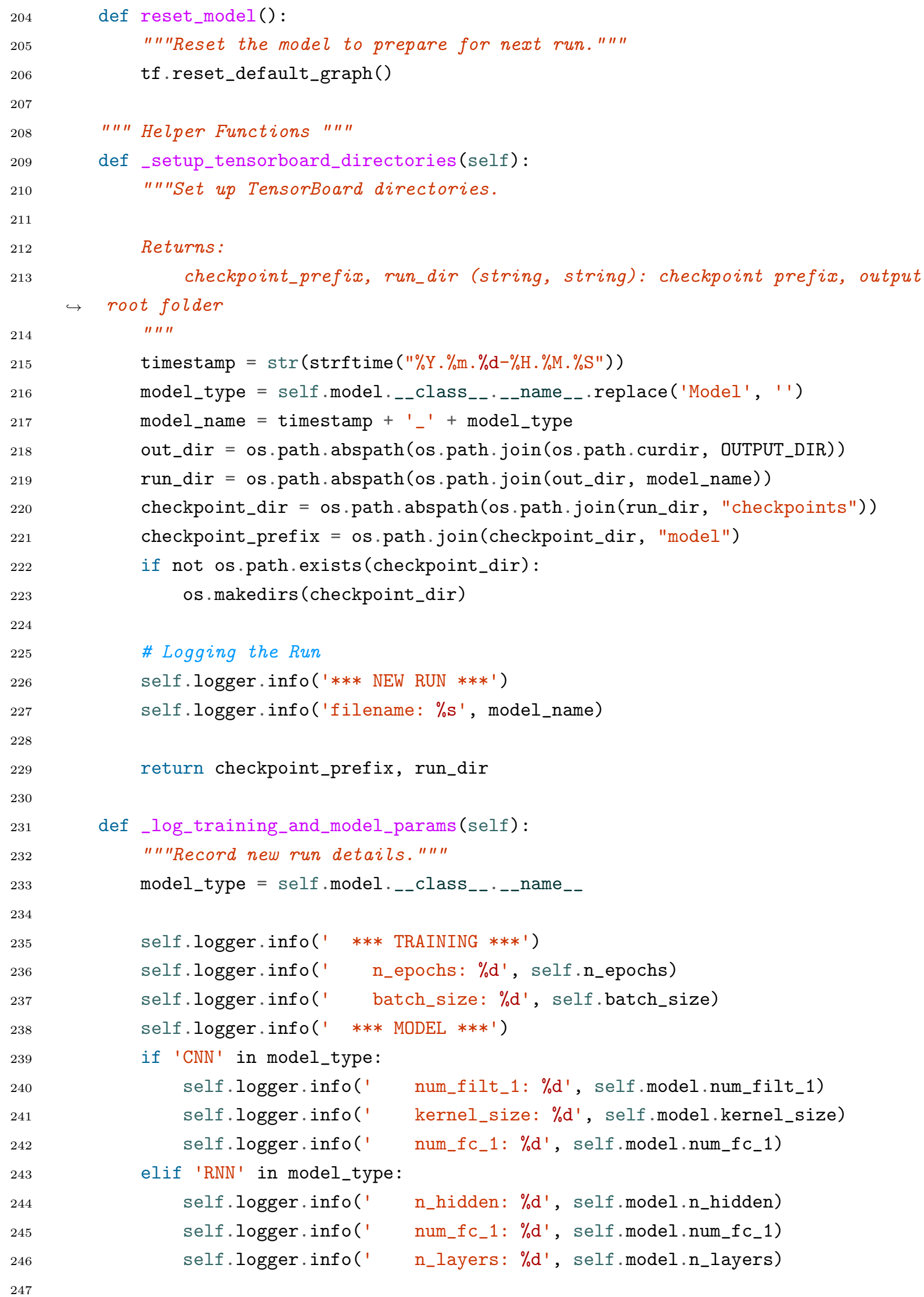









$\begin{array}{ll}292 & \text { \# create file handler which logs even debug messages } \\ 293 & \text { fh = logging.FileHandler(LOGFILE_NAME) } \\ 294 & \text { fh.setLevel(logging.INFO) } \\ 295 & \text { \# create console handler with a higher log level } \\ 296 & \text { ch = logging.StreamHandler() } \\ 297 & \text { ch.setLevel(logging.INFO) } \\ 298 & \text { \# create formatter and add it to the handlers } \\ 299 & \text { formatter = logging.Formatter(DEFAUL_FORMAT) } \\ 300 & \text { fh.setFormatter(formatter) } \\ 301 & \text { ch.setFormatter(formatter) } \\ 302 & \text { \#add the handlers to the logger } \\ 303 & \text { logger.addHandler(fh) } \\ 304 & \text { logger.addHandler(ch) } \\ 305 & \text { main() } \\ 306 & \end{array}$




\section{B.5 tune.py}

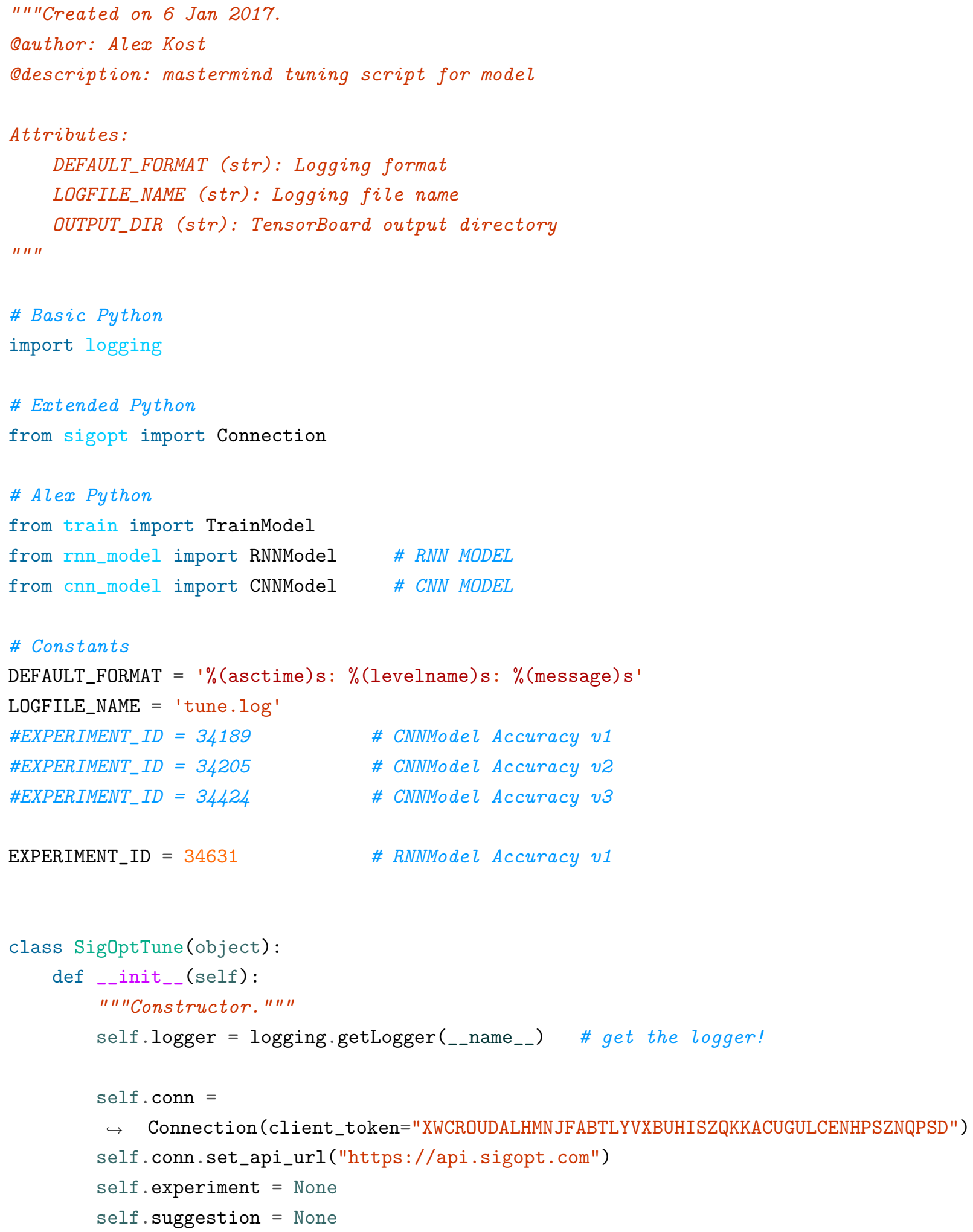


self.model $=$ None

self $\cdot$ acc $=$ None

def create_cnn_experiment(self):

""Create experiment. Modify as needed." "I"

self.experiment $=$ self $\cdot \operatorname{conn} \cdot$ experiments ()$\cdot \operatorname{create}($

name="CNNModel Accuracy v3",

parameters $=[\operatorname{dict}($ name $="$ learning_rate" , bounds $=\operatorname{dict}(\min =0.00001, \max =0.1)$, type="double"),

$\operatorname{dict}($ name="dropout_rate" , bounds $=\operatorname{dict}(\min =0.2, \max =0.9)$, type="double"),

$\operatorname{dict}($ name="beta1", bounds=dict $(\min =0.0001, \max =0.999)$, type="double"),

$\operatorname{dict}($ name="beta2" , bounds $=\operatorname{dict}(\min =0.0001, \max =0.999)$, type="double"), dict (name="epsilon", bounds=dict $(\min =1 \mathrm{e}-8, \max =1.0)$, type="double"), $\operatorname{dict}($ name="num_filt_1", bounds=dict $(\min =1, \max =40)$, type="int"), dict (name="kernel_size", bounds=dict $(\min =1, \max =10)$, type="int"), $\operatorname{dict}\left(\right.$ name $="$ num_f $_{-} c_{-} 1 "$, bounds=dict $(\min =1, \max =40)$, type="int")

])

self.logger.info('Experiment created! ID \%d.', self.experiment.id)

def create_rnn_experiment(self):

"" "Create experiment. Modify as needed.""

self.experiment $=$ self. conn. experiments () . create (

name="RNNModel Accuracy v1", parameters $=[$ dict (name="learning_rate" , bounds $=\operatorname{dict}(\min =0.00001, \max =0.1)$, type="double"), dict (name="dropout_rate", bounds $=\operatorname{dict}(\min =0.2, \max =0.9)$, 


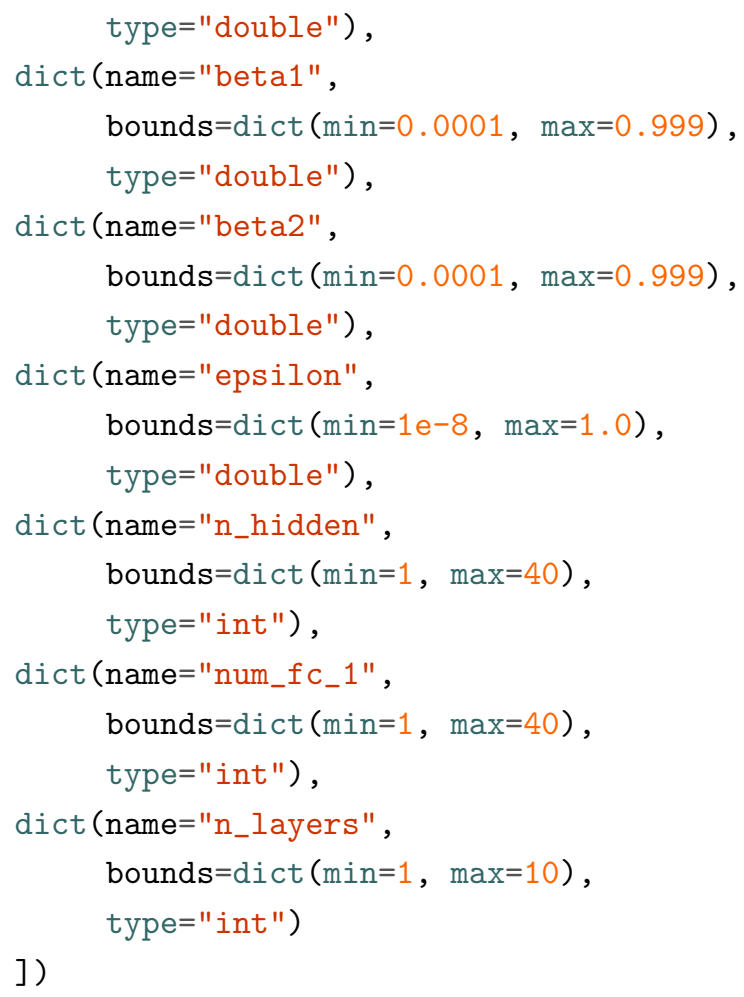




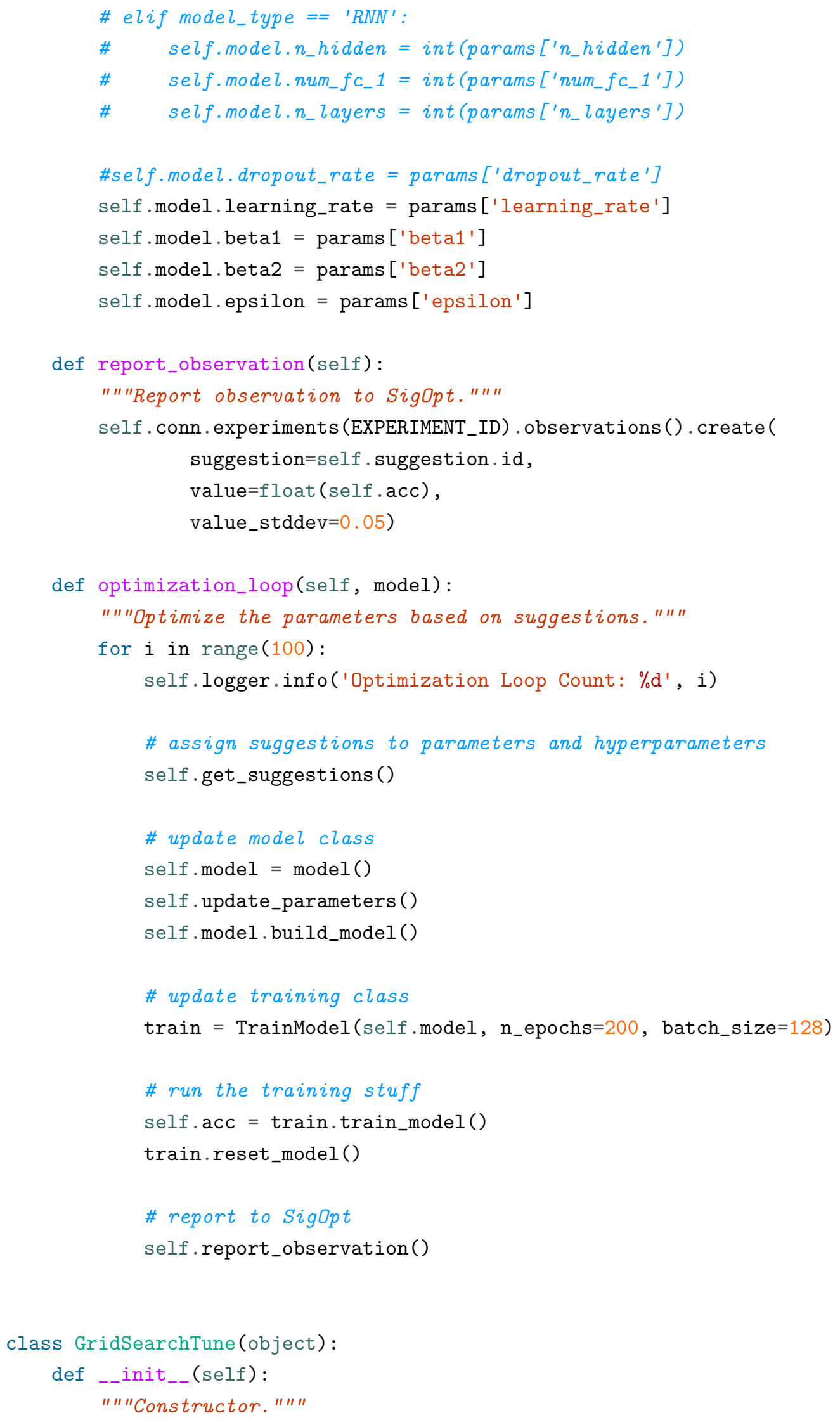




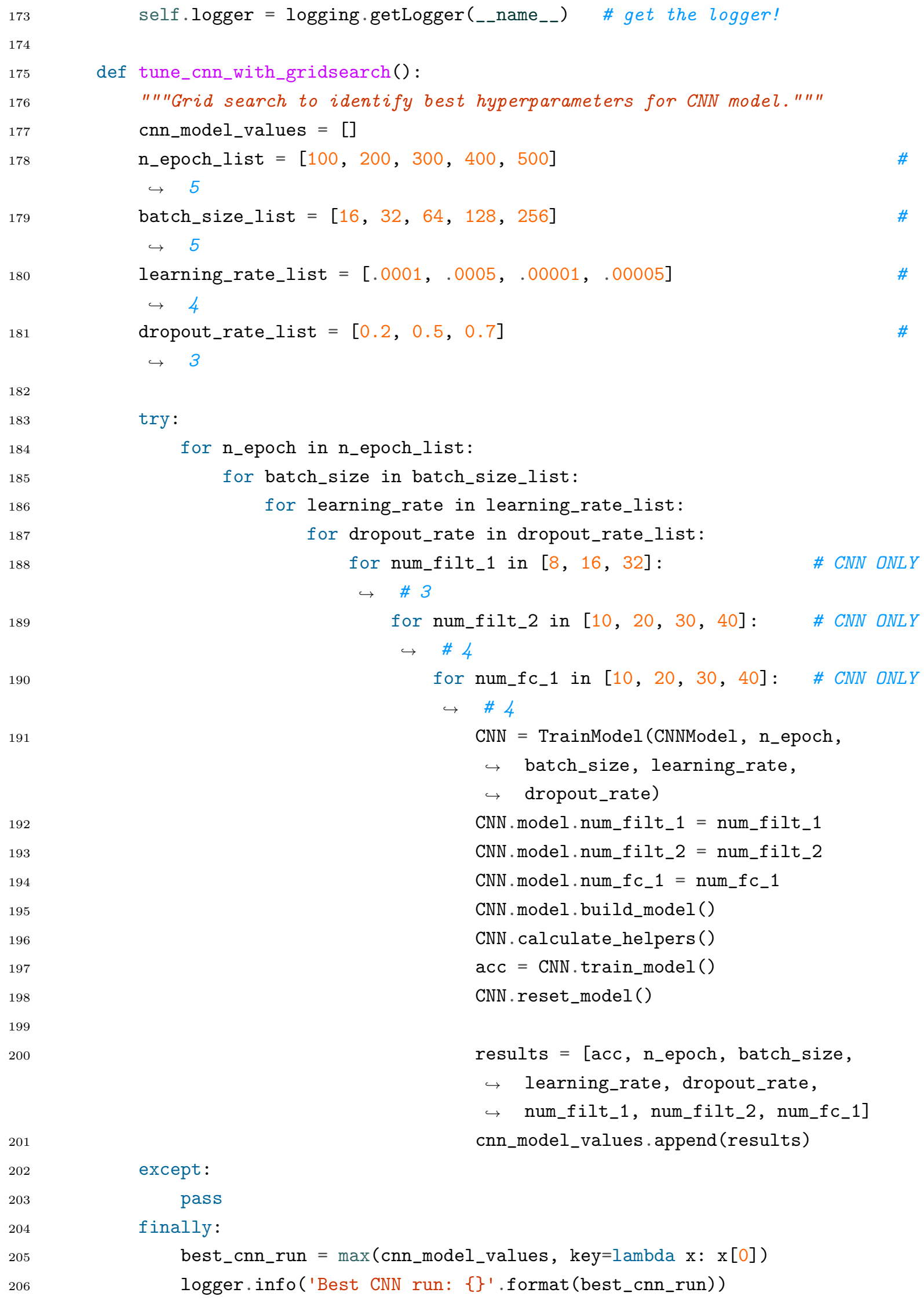




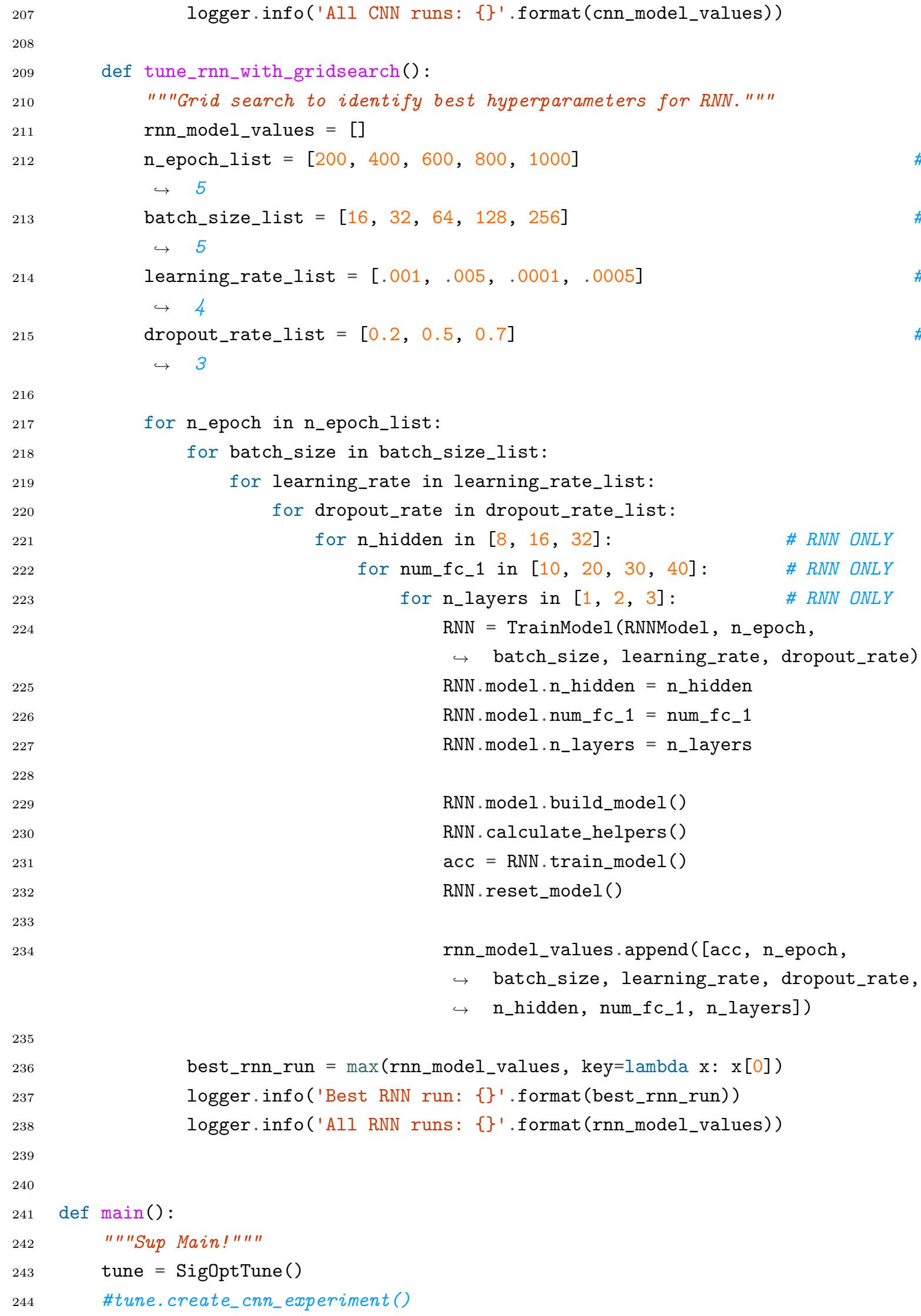









\section{B.6 main.m}

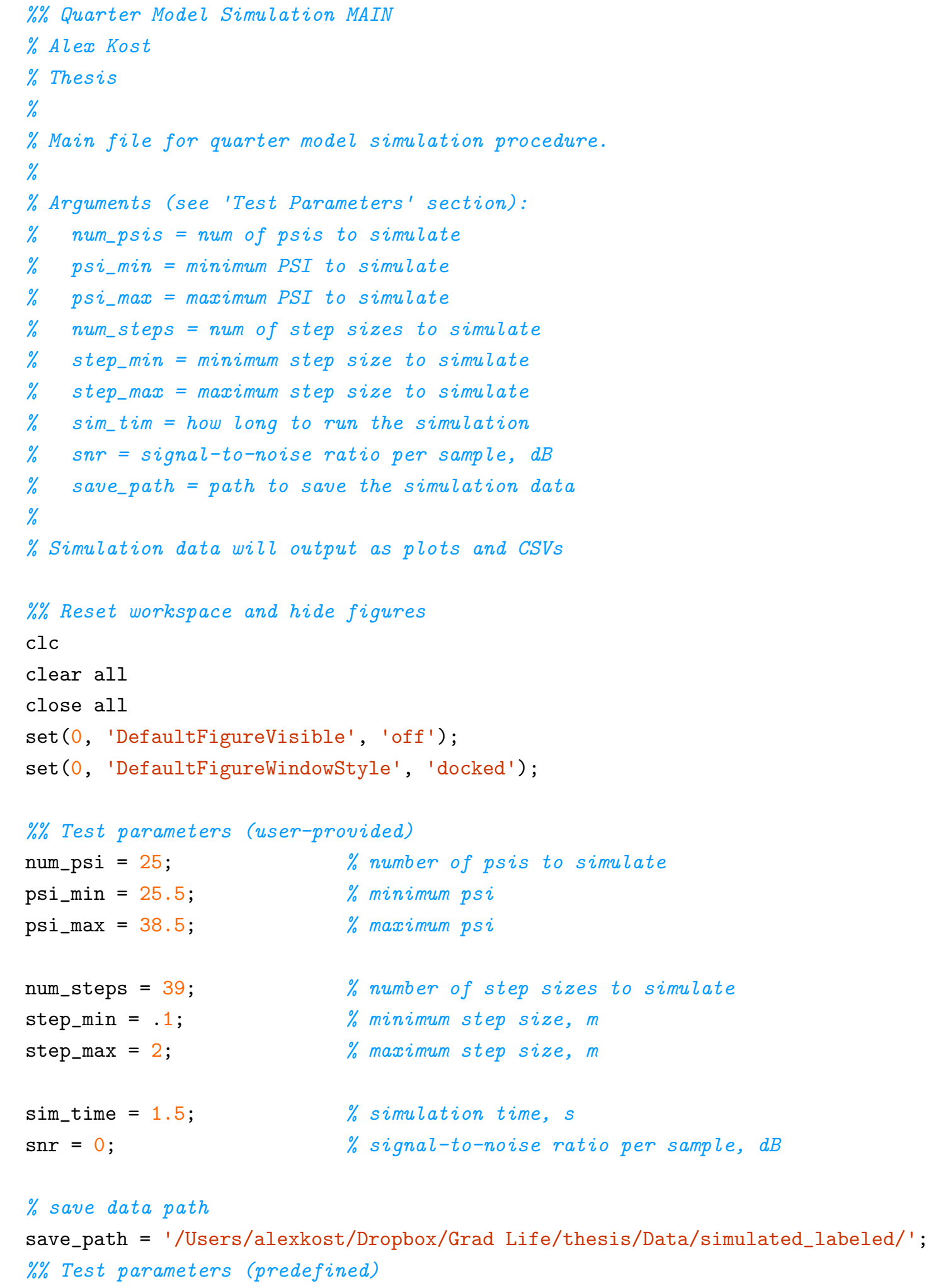




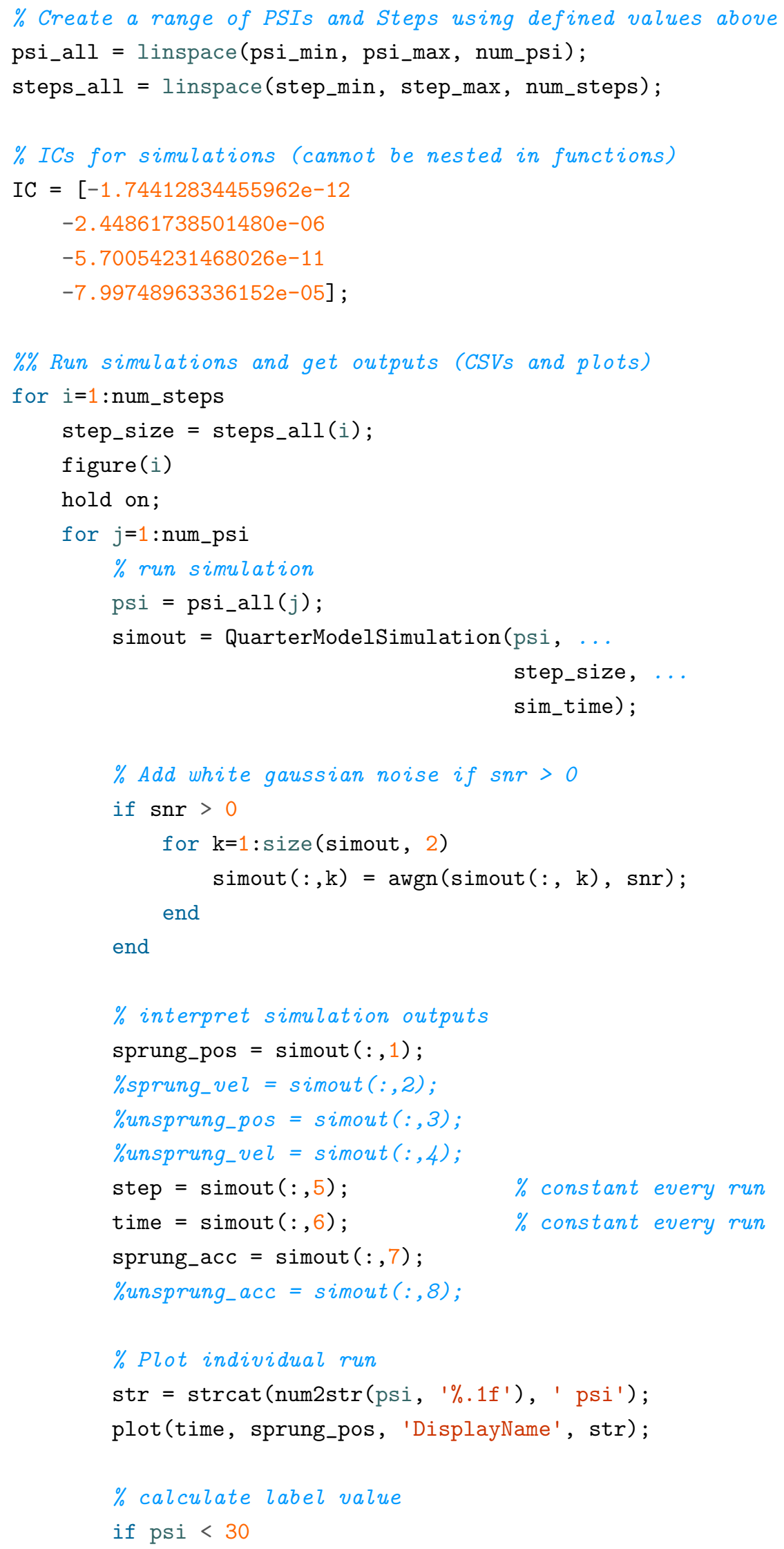


121 end label_val = 0 ;

elseif psi <= 34

label_val = 1 ;

elseif psi > 34

label_val = 2;

end

$\%$ Output to CSV

\% Modifications done for Tensorflow

$\%$ use sprung acceleration data only ( 1 feature)

$\% \quad$ transpose so each row is independent example

\% remove first .45 seconds of data

filename $=\operatorname{strcat}\left(\right.$ 'Sim_ $^{\prime}, \ldots$

num2str (psi, 1\%.1f'), 'psi_', ...



fullfilename $=$ fullfile(save_path, num2str (label_val), filename);

acc_transposed $=$ [sprung_acc] ' ;

$\mathrm{M}=$ acc_transposed $(:,(.45 / .001):$ end $)$;

label_val_column $=\operatorname{ones}(\operatorname{size}(\mathrm{M}, 1), 1) *$ label_val;

csvwrite(fullfilename, horzcat(label_val_column, M)) ;

end

$\%$ create figure with step

plot (time, step, '--', 'DisplayName', 'Step');

hold off;

title(sprintf('Quarter-Car Motion \nStep size = \% [m]', step_size)); xlabel ('Time (s)');

ylabel ('Vehicle height (m)');

legend ('show');

$\%$ save figure

filename $=\operatorname{sprintf}($ Plot_step_size_\%g.png', step_size);

fullfilename $=$ fullfile(save_path, filename);

print (figure (i), fullfilename, '-dpng ', '-r300'); 


\section{B.7 QuarterModelSimulation.m}

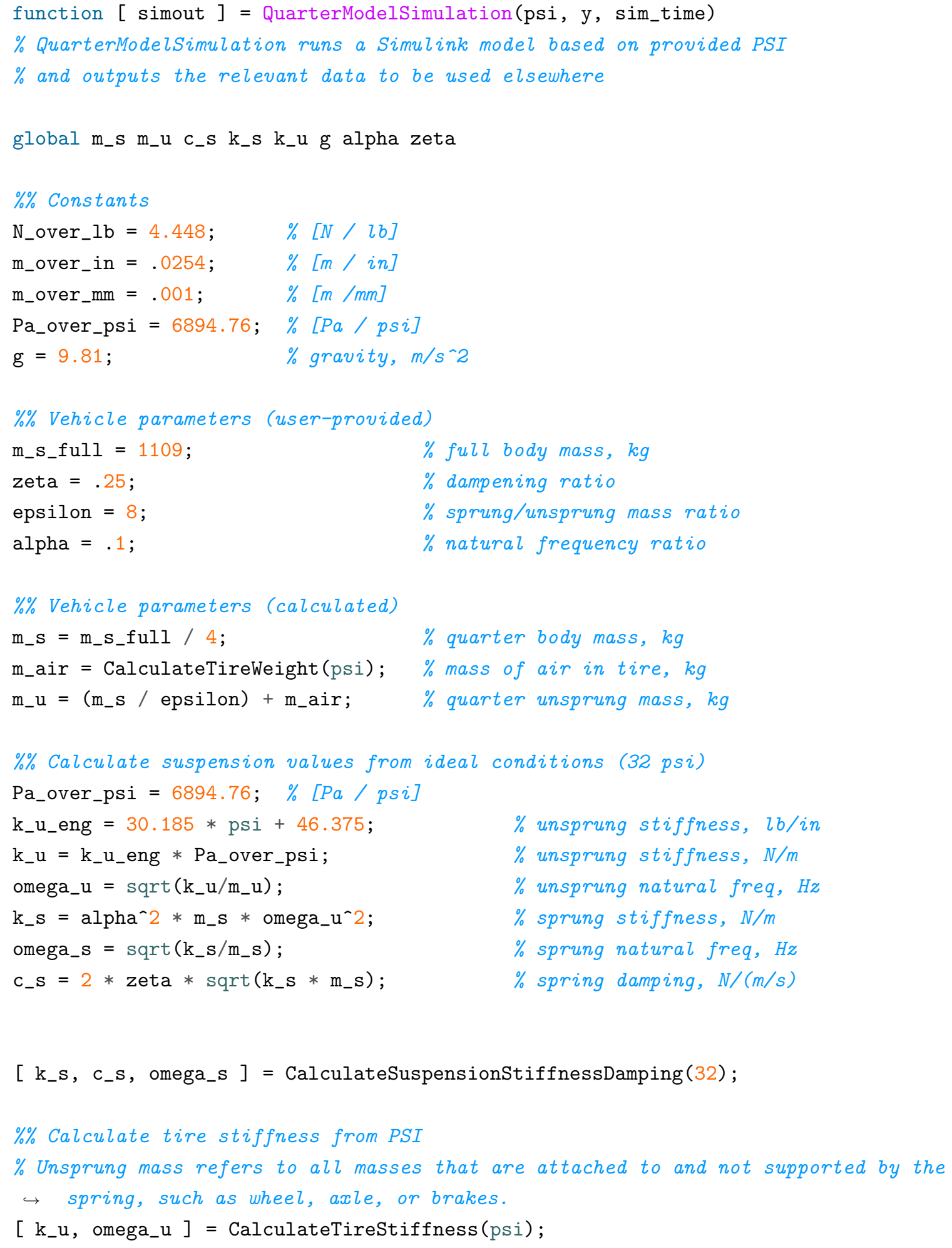




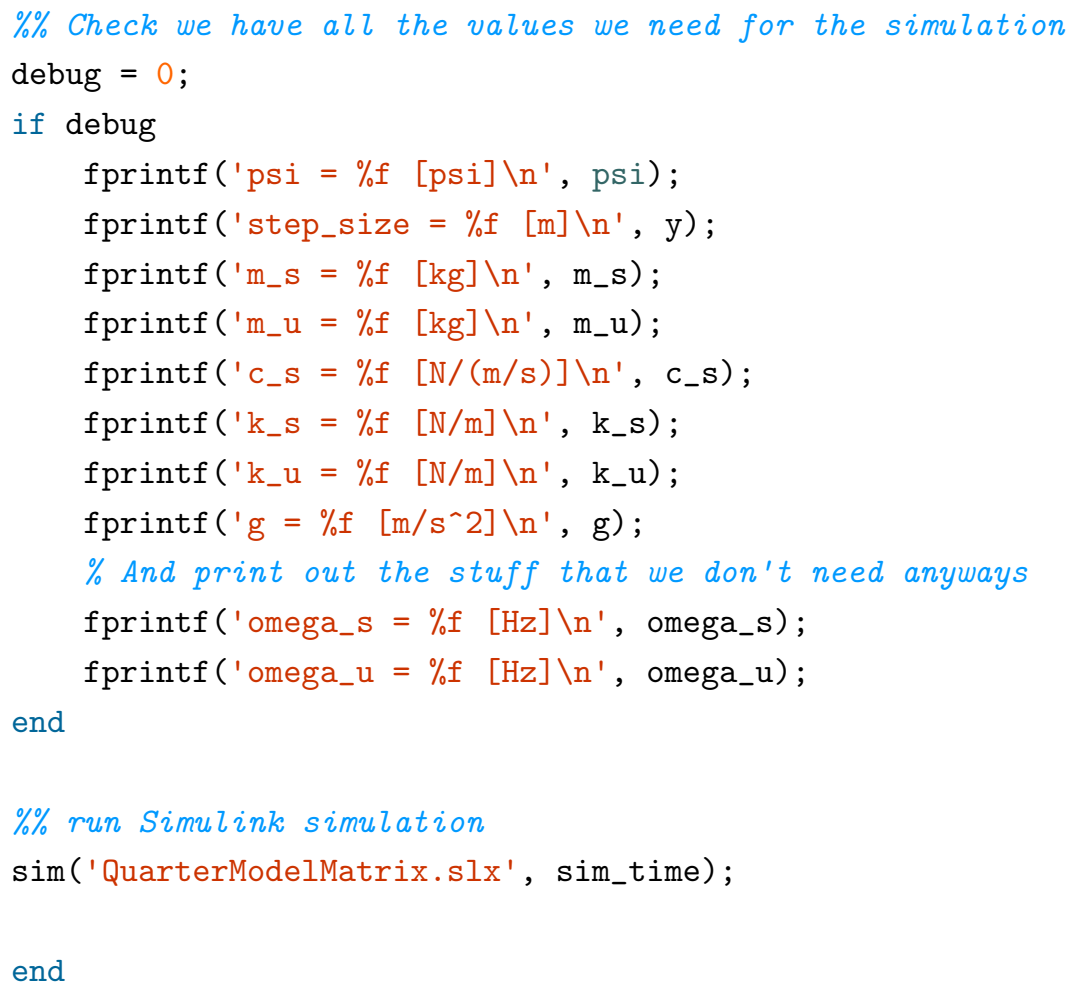




\section{B.8 CalculateSuspensionStiffnessDamping.m}

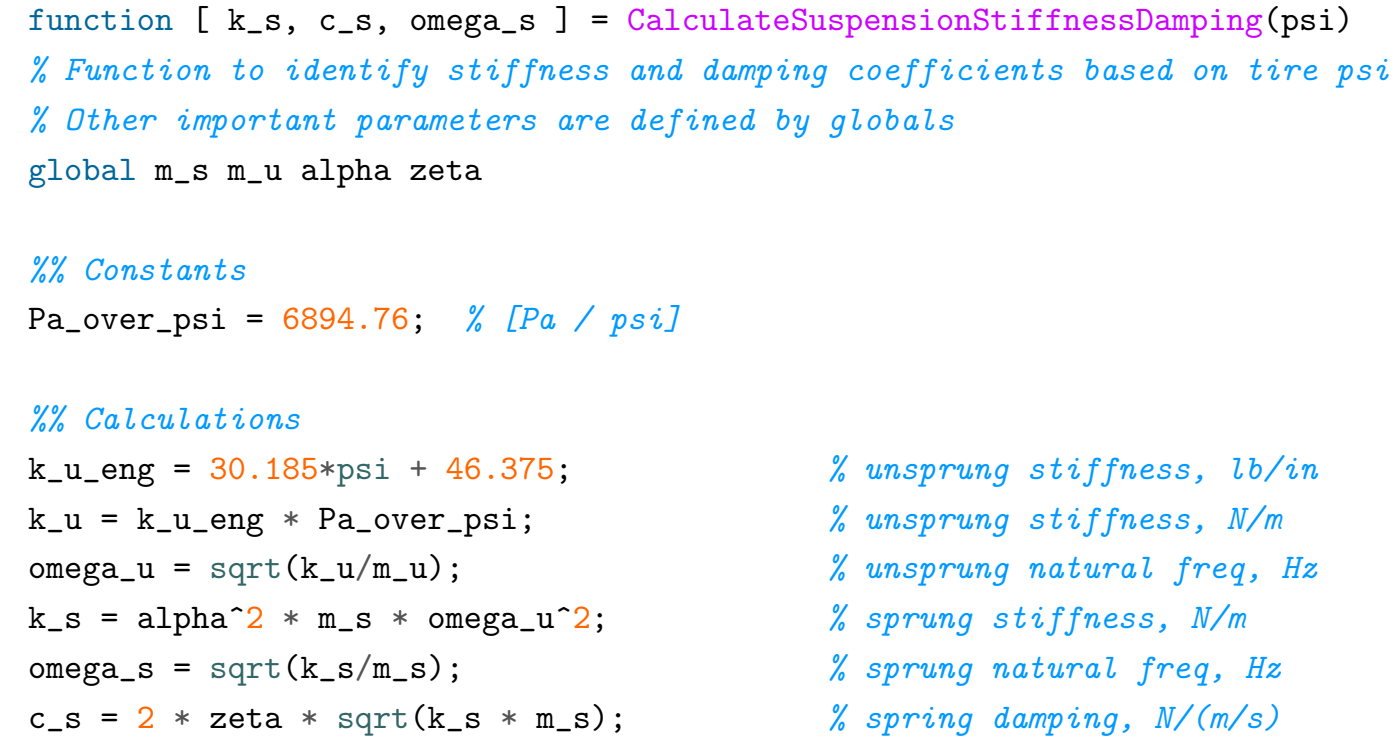




\section{B.9 CalculateTireStiffness.m}

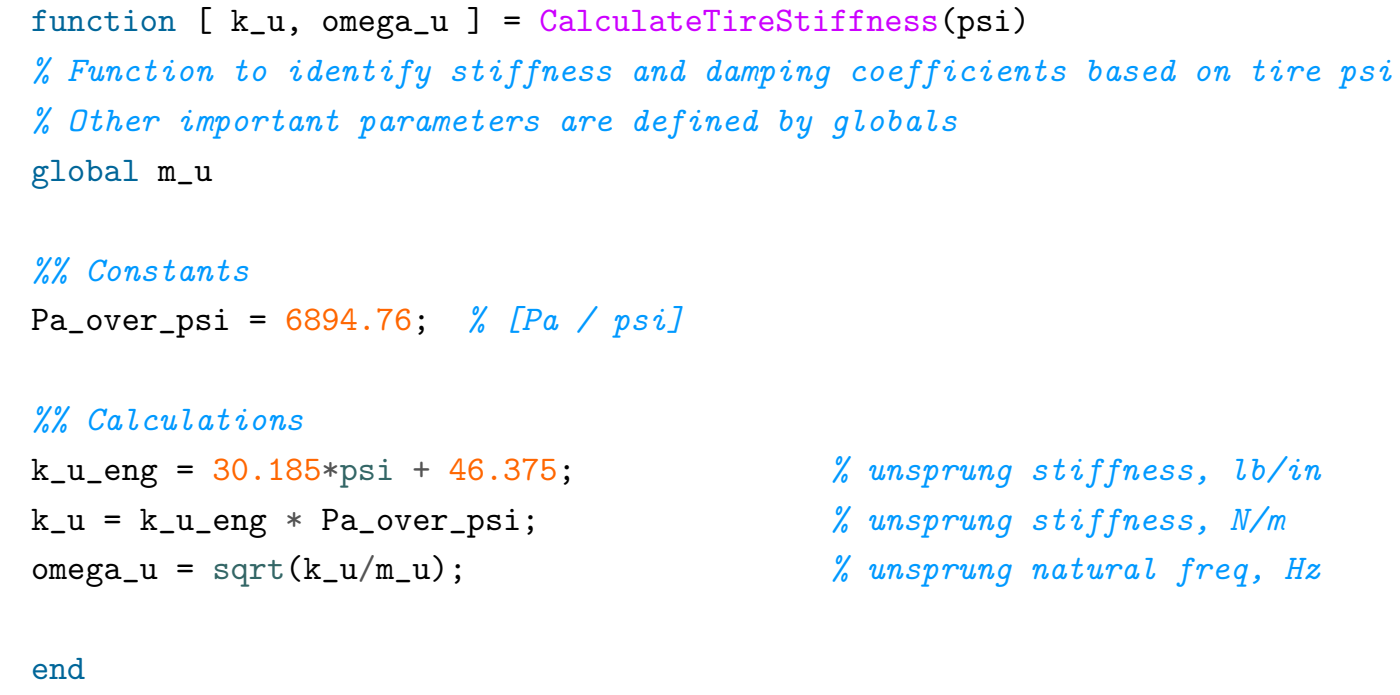




\section{B.10 SimFunc.m}

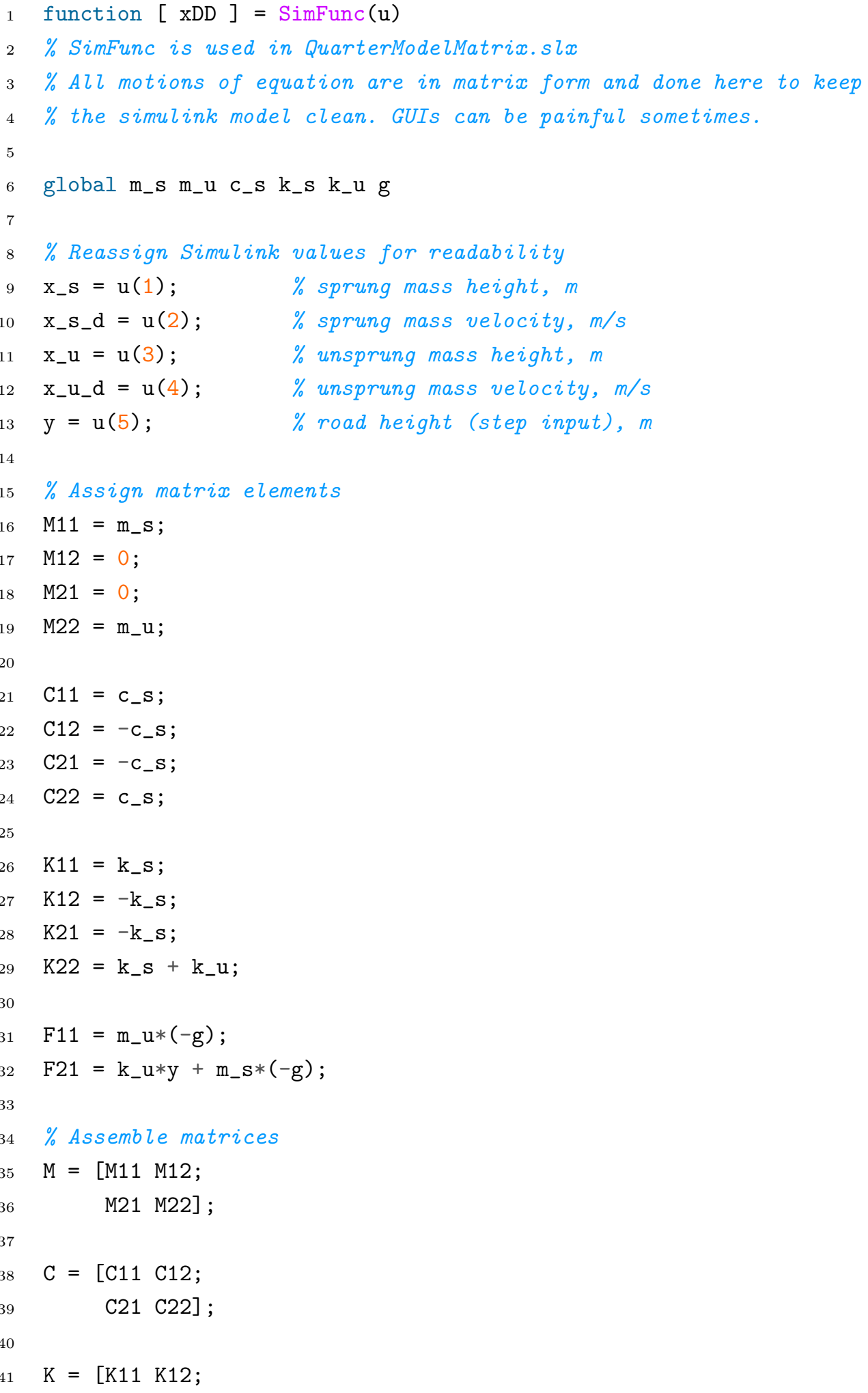







B.11 QuarterModelMatrix.slx



Figure B.1: QuarterModelMatrix.slx 